\title{
MEAN CONVERGENCE OF HERMITE AND LAGUERRE SERIES. II
}

\author{
BY \\ BENJAMIN MUCKENHOUPT( $\left.{ }^{1}\right)$
}

1. Introduction. The purpose of this paper is to prove inequalities of the form

$$
\left\|s_{n}(x) U(x)\right\|_{p} \leqq C\|f(x) V(x)\|_{p}
$$

where $s_{n}$ is the $n$th partial sum of a Hermite or Laguerre series for $f(x), U(x)$ and $V(x)$ are suitable weight functions and the norm is over $(-\infty, \infty)$ in the Hermite case and over $(0, \infty)$ in the Laguerre case. Mean convergence results of the type $\lim _{n \rightarrow \infty}\left\|\left[s_{n}(x)-f(x)\right] U(x)\right\|_{p}=0$ for every $f$ satisfying $f(x) V(x) \in L^{p}$ will be obtained as corollaries of these inequalities. For the convenience of readers interested primarily in the results, the main theorems proved are concentrated at the beginning of $\S \S 4$ and 5. Three other results showing that these theorems are in many ways the best possible are given at the beginning of $\S \S 6$ and 8 .

Usually inequalities of the type (1.1) are proved only when $U(x)=V(x)$; however, as shown in [4], no such theorem can be proved for Hermite and Laguerre series for $1 \leqq p \leqq 4 / 3$ or $p \geqq 4$ if $U(x)=V(x)$. Surprisingly, $U(x)$ and $V(x)$ in the results obtained, although they contain exponential factors, need never differ by more than a factor of $(1+|x|)^{1 / 3}$ in the Hermite case and $(1+x)^{1 / 6}$ in the Laguerre case. In the basic theorems proved for $1<p<\infty$, Theorems 1 and $7, U(x)$ can always be taken equal to $V(x)$ if $4 / 3<p<4$ and the minimum ratio of $V(x)$ to $U(x)$ increases as $p$ moves away from the interval $(4 / 3,4)$. It is also possible to make $U(x)=V(x)$ even for $1<p \leqq 4 / 3$ and $4 \leqq p<\infty$ if a small part of the integral of $s_{n} U$ or the integral defining $s_{n}$ is omitted; details of this are given in Theorems 3, 4, 9 and 10. Substitute results, Theorems 2, 5, 8 and 11, are also obtained for $p=1$; as usual these theorems require that $|f(x)|\left(1+\log ^{+}|f(x)|\right)$ be integrable with a suitable weight function. Here $\log ^{+} x$ is the function that equals $\log x$ for $x \geqq 1$ and is 0 otherwise.

The theorems proved here are a generalization of the results of Askey and Wainger in [1] in several ways. Their result was to prove inequalities of the form (1.1) with $4 / 3<p<4$ and $U(x)=V(i)=\exp \left(-\frac{1}{2} x^{2}\right)$ in the Hermite case and $U(x)=V(x)=e^{-x / 2} x^{\alpha / 2}$ in the case of Laguerre series of order $\alpha \geqq 0$. The results here are valid for $1 \leqq p<\infty$; for Laguerre series they are valid for all $\alpha>-1$; and in the cases considered by Askey and Wainger it is shown that other functions can be used as the weight function.

Received by the editors April 1, 1969.

( $\left.{ }^{1}\right)$ Supported in part by NSF grant GP 7539.

Copyright (C) 1970, American Mathematical Society 
In the Hermite case the functions $U(x)$ and $V(x)$ in (1.1) will be taken to have the form $w(x, b)=(1+|x|)^{b} \exp \left(-\frac{1}{2} x^{2}\right)$; in a few cases $V(x)$ will also contain a factor of $1+\log ^{+} x$. The justification for considering only these weight functions is Theorem 13. It is shown there that if (1.1) holds and $U(x)$ is not zero on a set of positive measure, then there is a constant, $C_{1}$, such that for every $t \geqq 1$

$$
\int_{-t}^{t}\left|U(x) \exp \left(\frac{1}{2} x^{2}\right)\right|^{p} d x \leqq C_{1} t^{p}
$$

and

$$
\int_{-t}^{t}\left|V(x) \exp \left(\frac{1}{2} x^{2}\right)\right|^{-q} d x \leqq C_{1} t^{q}
$$

where $1 / p+1 / q=1$. It will also be assumed that $U$ and $V$ are bounded away from 0 and $\infty$ for $x$ near 0 ; this is certainly desirable and the nature of the weight function for Hermite polynomials, $\exp \left(-x^{2}\right)$, suggests that this should be possible. The largest function of a reasonable form that satisfies this condition and (1.2) is $w(x, 1-1 / p)$. Similarly, the smallest function of a reasonable form that satisfies , this condition and (1.3) is $w(x,-1 / p)$. Since it is also desirable to keep $U$ and $V$ as close to each other as possible, $w(x, 1-1 / p)$ and $w(x,-1 / p)$ should be close to being the upper and lower bounds for both $U$ and $V$. The only "reasonable" functions that fit all these criteria for $U$ and $V$ are of the form $w(x, b)\left(1+\log ^{+} x\right)^{\beta}$. Because of the complications involved, the logarithmic factors will not be used except in a few cases where they are necessary to produce the most "natural" theorem. In these exceptional cases, if $U(x)=w(x, b)$ and $V(x)=w(x, B)$, a strict inequality would relate $b$ and $B$ instead of a more desirable weak inequality.

Similar considerations based on Theorem 14 show that for the Laguerre case the only "natural" weight functions are of the form

$$
(x /(1+x))^{a}(1+x)^{b}\left(1+\log ^{+} x\right)^{\beta} e^{-x / 2} x^{\alpha / 2} .
$$

Again, the log terms will be used in only a few cases.

In $\S 2$ various facts about Laguerre and Hermite polynomials are stated; these are either from [1] or are extensions of the results there. $\$ 3$ is concerned with weighted inequalities for Hilbert transforms. Weighted forms of Hardy's inequality and the usual Hilbert transform theorem are used to obtain the necessary inequalities. $\$ \S 4$ and 5 contain the main theorems; it is surprising that even in the most complicated Laguerre proof only ten distinct integrals are estimated. Of these, five are weighted Hilbert transform expressions of the type treated in $\$ 3$; for three Hölder's inequality is used; and for two a simple estimation works. $\S 6$ contains the theorems mentioned above that show the weight functions considered are the only reasonable ones that could work. $\$ 7$ contains results about Laguerre and Hermite polynomials that permit the estimation of lower bounds for various integrals containing them. These are used in $\$ 8$ to show that, except for one peculiar case, the results obtained in $\$ \S 3$ and 4 are the best possible for weight functions of the given form. 
2. Facts about Laguerre and Hermite polynomials. As usual, the Hermite polynomials, $H_{n}(x)$, will be defined by $\sum H_{n}(x) r^{n} / n !=\exp \left(2 x r-r^{2}\right)$ and for $\alpha>-1$ the Laguerre polynomials will be defined by

Following [1], the functions

$$
\sum L_{n}^{\alpha}(x) r^{n}=(1-r)^{-\alpha-1} \exp \left(\frac{-r x}{1-r}\right)
$$

$$
\mathscr{H}_{n}(x)=\exp \left(-\frac{1}{2} x^{2}\right)\left(\pi^{1 / 2} 2^{n} n !\right)^{-1 / 2} H_{n}(x)
$$

and

$$
\mathscr{L}_{n}^{\alpha}(x)=\left(\frac{n !}{\Gamma(n+\alpha+1)}\right)^{1 / 2} x^{\alpha / 2} e^{-x / 2} L_{n}^{\alpha}(x)
$$

will be used since they have simpler estimates than $H_{n}(x)$ and $L_{n}^{\alpha}(x)$.

Using the table on p. 700 of [1] shows that there are positive constants, $C$ and $\gamma$, independent of $x$ and $n$, such that for every integer, $n \geqq 0$,

$$
\begin{aligned}
\left|\mathscr{H}_{n}(x)\right| & \leqq C\left(N^{1 / 3}+\left|x^{2}-N\right|\right)^{-1 / 4}, & & x^{2} \leqq 2 N, \\
& \leqq C \exp \left(-\gamma x^{2}\right), & & x^{2}>2 N,
\end{aligned}
$$

where $N=2 n+1$. The same table also gives the estimates

$$
\begin{aligned}
\left|\mathscr{H}_{n+1}(x)-\mathscr{H}_{n-1}(x)\right| & \leqq C N^{-1 / 2}\left(N^{1 / 3}+\left|x^{2}-N\right|\right)^{1 / 4}, & & x^{2} \leqq 2 N, \\
& \leqq C \exp \left(-\gamma x^{2}\right), & & x^{2}>2 N
\end{aligned}
$$

for all integers, $n \geqq 1$.

The table on p. 699 of [1] shows that, given an $\alpha \geqq 0$, there are positive constants, $C$ and $\gamma$,independent of $x$ and $n$, such that for all integers, $n \geqq 0$,

$$
\begin{array}{rlrl}
\left|\mathscr{L}_{n}^{\alpha}(x)\right| & \leqq C x^{\alpha / 2} \nu^{\alpha / 2}, & 0 \leqq x \leqq 1 / \nu \\
& \leqq C x^{-1 / 4} \nu^{-1 / 4}, & 1 / \nu & <x \leqq \nu / 2 \\
& \leqq C \nu^{-1 / 4}\left(\nu^{1 / 3}+|x-\nu|\right)^{-1 / 4} & \nu / 2 & <x \leqq 3 \nu / 2 \\
& \leqq C e^{-\gamma x}, & 3 \nu / 2 & <x
\end{array}
$$

where $\nu=4 n+2 \alpha+2$. Similarly, for $n \geqq 1$,

$$
\begin{aligned}
\left|\mathscr{L}_{n+1}^{\alpha}(x)-\mathscr{L}_{n-1}^{\alpha}(x)\right| & \leqq C x^{\alpha / 2} \nu^{\alpha / 2-1}, & 0 \leqq x \leqq 1 / \nu, \\
& \leqq C x^{1 / 4} \nu^{-3 / 4}, & 1 / \nu<x \leqq \frac{1}{2} \nu, \\
& \leqq C \nu^{-3 / 4}\left(\nu^{1 / 3}+|x-\nu|\right)^{1 / 4}, & \nu / 2<x \leqq 3 \nu / 2, \\
& \leqq C e^{-\gamma x}, & 3 \nu / 2<x .
\end{aligned}
$$

It will now be shown that (2.5) and (2.6) are also valid for $-1<\alpha<0$ with the proviso that if $n=0$ and $\alpha$ is negative, then $\nu$ should be taken equal to 2 in (2.5). Using the differential equation for $L_{n}^{\alpha}(x),(5.1 .2)$, p. 99 of [5], the fact that $d L_{n}^{\alpha}(x) / d x=-L_{n-1}^{\alpha+1}(x),(5.1 .14)$, p. 101 of [5], and (2.2) shows that

$$
\mathscr{L}_{n}^{\alpha}(x)=-((n-1) / n)^{1 / 2} \mathscr{L}_{n-2}^{\alpha+2}(x)+\left((\alpha+1-x) / n^{1 / 2} x^{1 / 2}\right) \mathscr{L}_{n-1}^{\alpha+1}(x) .
$$


Using (5.1.13), p. 101 of [5] and (2.2) shows that

$$
\mathscr{L}_{n}^{\alpha}(x)=((n+\alpha+1) / x)^{1 / 2} \mathscr{L}_{n}^{\alpha+1}(x)-(n / x)^{1 / 2} \mathscr{L}_{n-1}^{\alpha+1}(x) .
$$

For $0 \leqq x \leqq \frac{1}{2} \nu,(2.7)$ combined with (2.5) for $\alpha \geqq 0$ will prove (2.5) for $-1<\alpha<0$. Similarly, for $x>\frac{1}{2} \nu,(2.8)$ can be used to prove (2.5) for $-1<\alpha<0$. That (2.6) is valid for $-1<\alpha<0$ follows similarly from (2.7) and (2.8); for this various facts of the type $(n+1)^{1 / 2}=n^{1 / 2}+O\left(n^{-1 / 2}\right)$ must be used.

As usual for partial sum theorems, expressions for the relevant Poisson kernels will be needed. Now define

$$
d_{n}(x, y)=\sum_{k=0}^{n} \mathscr{H}_{k}(x) \mathscr{H}_{k}(y) .
$$

Using (5.5.9), p. 105 of [5] and (2.1) shows that

$$
d_{n}(x, y)=\left(\frac{n+1}{2}\right)^{1 / 2} \frac{\mathscr{H}_{n+1}(x) \mathscr{H}_{n}(y)-\mathscr{H}_{n}(x) \mathscr{H}_{n+1}(y)}{x-y} .
$$

Now let

$$
\begin{gathered}
h_{1}(n, x, y)=\mathscr{H}_{n}(x) \mathscr{H}_{n}(y), \\
h_{2}(n, x, y)=\frac{n^{1 / 2} \mathscr{H}_{n}(y)\left[\mathscr{H}_{n+1}(x)-\mathscr{H}_{n-1}(x)\right]}{x-y}, \\
h_{3}(n, x, y)=h_{2}(n, y, x)
\end{gathered}
$$

and $a_{n}=((n+1) / 2)^{1 / 2}$. Then, following the procedure of p. 702 of [1],

$$
d_{n}=\frac{1}{2}\left(d_{n}+d_{n-1}+h_{1}\right)=\frac{a_{n-1}}{2}\left[\frac{d_{n}}{a_{n}}+\frac{d_{n-1}}{a_{n-1}}\right]+\left[\frac{a_{n}-a_{n-1}}{2 a_{n}}\right] d_{n}+\frac{1}{2} h_{1} .
$$

Replacing the first term in parentheses on the right side of (2.14) by $n^{-1 / 2}\left(h_{2}+h_{3}\right)$, shifting the second main term to the far left side, and then dividing by the resulting coefficient of $d_{n}$ on the left, shows that

$$
d_{n}=b_{n} h_{1}+c_{n}\left(h_{2}+h_{3}\right)
$$

where $b_{n}$ and $c_{n}$ are functions of $n$ bounded above by 1 and below by $1 / 3$. This shows that for any function, $f(x)$, and any set, $E$,

$$
\left|\int_{E} d_{n}(x, y) f(y) d y\right| \leqq \sum_{k=1}^{3}\left|\int_{E} h_{k}(n, x, y) f(y) d y\right|
$$

where the singular integrals are taken in the principal value sense. This will be the basis for the proofs concerning mean convergence of Hermite series.

Similarly, given a fixed $\alpha>-1$, define

$$
D_{n}(x, y)=\sum_{k=0}^{n} \mathscr{L}_{k}^{\alpha}(x) \mathscr{L}_{k}^{\alpha}(y),
$$




$$
\begin{aligned}
& j_{1}(n, x, y)=\mathscr{L}_{n}^{\alpha}(x) \mathscr{L}_{n}^{\alpha}(y), \\
& j_{2}(n, x, y)=\frac{n \mathscr{L}_{n}^{\alpha}(y)\left[\mathscr{L}_{n+1}^{\alpha}(x)-\mathscr{L}_{n-1}^{\alpha}(x)\right]}{y-x},
\end{aligned}
$$

and

$$
j_{3}(n, x, y)=j_{2}(n, y, x) .
$$

Using (5.1.11), p. 100 of [5] and (2.2) then shows that

$$
D_{n}(x, y)=(n+1)^{1 / 2}(n+\alpha+1)^{1 / 2} \frac{\mathscr{L}_{n}^{\alpha}(x) \mathscr{L}_{n+1}^{\alpha}(y)-\mathscr{L}_{n+1}^{\alpha}(x) \mathscr{L}_{n}^{\alpha}(y)}{x-y} .
$$

Following the same procedure as above shows that

$$
D_{n}=b_{n} j_{1}+c_{n}\left(j_{2}+j_{3}\right)
$$

where $b_{n}$ and $c_{n}$ are bounded above and below by positive constants depending only on $\alpha$.

The following density results will be needed.

LEMMA 1. If $1 \leqq p<\infty$ and $a$ and $b$ are real, then the functions,

$$
x^{a}(1+x)^{b} \exp \left(-\frac{1}{2} x\right) x^{n}, \quad n=0,1,2, \ldots,
$$

are dense in $L^{p}(0, \infty)$.

LEMMA 2. If $1 \leqq p<\infty$ and $b$ is real, then the functions, $(1+|x|)^{b} \exp \left(-\frac{1}{2} x^{2}\right) x^{n}$, $n=0,1,2, \ldots$, are dense in $L^{p}(-\infty, \infty)$.

The proof for Lemma 1 is similar to the proofs of Theorems 5.7.1-5.7.3, pp. $107-109$ of [5]. It may be assumed that $a \geqq 0$, for if it were not, the functions with negative powers of $x$ could be omitted and the theorem proved for the remaining functions. Then let $\phi(x)=x^{a}(1+x)^{b} \exp \left(-\frac{1}{2} x\right)$ and $d \mu(y)=y^{-1}[\phi(-\log y)]^{p} d y$. Theorem 3.1 .5 , p. 40 of [5] shows that polynomials are dense in $L_{\mu}^{p}(0,1)$ and a change of variables shows that the functions $\phi(x) e^{-n x}$ are dense in $L^{p}(0, \infty)$. Following the argument of p. 108 of [5], it is sufficient for the proof of Lemma 6 to show that $\int_{0}^{\infty}\left|\phi(x) \sum_{n=0}^{\infty} L_{n}^{\alpha}(x) r^{n}\right|^{p} d x$ is finite for $0 \leqq r<1$ and $\alpha=0$. Using Minkowski's inequality and (2.2), it is sufficient to show that

$$
\sum_{n=0}^{\infty}\left(r^{n} \int_{0}^{\infty}\left|e^{x / 2} \phi(x) \mathscr{L}_{n}^{0}(x)\right|^{p} d x\right)^{1 / p}
$$

is finite for $0 \leqq r<1$. Using the estimates (2.5), the integral inside the sum is easily seen to be bounded above by a constant times $n$ to a fixed power; this is sufficient to give the desired convergence.

To prove Lemma 2 it is first shown that the functions $\left(1+|x|^{1 / 2}\right)^{b} \exp \left(-\frac{1}{2} x\right) x^{n}$ are dense in $L^{p}(0, \infty)$; this follows immediately from Lemma 1 . Then the reasoning used to prove the Hermite part of Theorem 5.7.1 of [5] can be applied. 
3. General inequalities. In this section some basic inequalities, Lemmas 3-7, are combined into results that will be used repeatedly in the proofs of the theorems in $\$ \S 4$ and 5. Lemmas 3-6 are variants of the well-known Hardy inequality, and Lemma 7 is a variant of the usual Hilbert transform theorem. Lemma 8 and its $L^{1}$ version, Lemma 9, are the most useful results in proving Theorems $1-12$. If $4 / 3<p<4$, Lemma 8 and Hölder's inequality are all that is needed for the proofs. If $p$ is not in the interval $(4 / 3,4)$, one integral arises in the proof of Theorems 1 and 7 that is of the form treated in Lemma 8 but with the parameters outside the range of validity of Lemma 8 . A preliminary for the estimation of this integral is given in Lemma 10; the results that are needed are given in Lemmas 11-13.

Throughout this paper, the singular integrals are to be taken in the principal value sense. The letter, $C$, will be used to denote constants, not necessarily the same at each occurrence. To simplify various expressions, the notation

$$
x^{r, s}=|x|^{r}(1+|x|)^{s-r}
$$

will be used.

LEMmA 3. If $1<p<\infty, r \geqq R, R<-1, s \leqq S, s<-1$ and $F(x)=\int_{0}^{x} f(y) d y$, then there is a constant, $C$, independent of $f$, such that

$$
\int_{0}^{\infty} x^{r, s}|F(x)|^{p} d x \leqq C \int_{0}^{\infty} x^{p} x^{R, s}|f(x)|^{p} d x .
$$

LEMmA 4. If $1<p<\infty, r \geqq R, r>-1, s \leqq S, S>-1$ and $F(x)=\int_{x}^{\infty} f(y) d y$, then there is a constant, $C$, independent of $f$, such that

$$
\int_{0}^{\infty} x^{r, s}|F(x)|^{p} d x \leqq C \int_{0}^{\infty} x^{p} x^{R, S}|f(x)|^{p} d x .
$$

Lemmas 3 and 4 are proved in the same way as the usual version of Hardys' inequality, (9.16), Vol. I, p. 20 of [7].

The stated conditions in Lemmas 3 and 4 are also sufficient to prove an $L^{1}$ inequality. However, since the final $L^{1}$ results will have $\log$ terms on the right, it is natural to prove a version of Lemmas 3 and 4 that contain log terms on the right. This allows slightly more liberal conditions on the exponents; the exact statements are Lemmas 5 and 6.

LemmA 5. If $r \geqq R, R \leqq-1, s \leqq S, s<-1$ and $F(x)=\int_{0}^{x} f(y) d y$, then there is $a$ constant, $C$, independent of $f$, such that

$$
\int_{0}^{\infty} x^{r, s}|F(x)| d x \leqq C+C \int_{0}^{\infty} x^{R, s} x|f(x)|\left(1+\log ^{+}|f(x)|\right) d x .
$$

Lemma 6. If $r \geqq R, r>-1, s \leqq S, S \geqq-1$ and $F(x)=\int_{x}^{\infty} f(y) d y$, then there is a constant, $C$, independent of $f$ such that

$$
\int_{0}^{\infty} x^{r, s}|F(x)| d x \leqq C+C \int_{0}^{\infty} x^{R, s} x|f(x)|\left(1+\log ^{+} x\right) d x .
$$


Lemma 5 is proved by assuming that $f(x)$ is nonnegative, reversing the order of integration on the left, and performing the inner integration. Except for the case $r=R=-1$, the result follows immediately. If $r=R=-1$, the troublesome part of the integral is

$$
-\int_{0}^{1} f(y) \log y d y .
$$

Now $g(x)=2 x\left(\log ^{+} x\right)$ and $h(x)=x$ for $x \leqq 2$ and $2 \exp \left(\frac{1}{2} x-1\right)$ for $x>2$ are conjugate in the sense of Young (see [7, Vol. I, p. 16]). Using Young's inequality, (3.1) is bounded by the sum of $\int_{0}^{1} g(f(x)) d x$ and $\int_{0}^{1} h(-\log y) d y$. This sum has the asserted bound.

Lemma 6 is also proved by interchanging the order of integration on the left; there are no troublesome cases in this proof.

LEMMA 7. Let $w(x)$ be a positive function and $B$ a constant and assume that for any integer, $n$, and $x$ in $\left[2^{n}, 2^{n+1}\right] w\left(2^{n}\right) \leqq B w(x) \leqq B^{2} w\left(2^{n}\right)$. If $\hat{f}(x)=\int_{x / 2}^{3 x / 2}[f(y) /(x-y)] d y$, then there is a constant, $C$, depending only on $p$ and $B$, such that

$$
\begin{array}{rlr}
\int_{0}^{\infty}|f(x)|^{p} w(x) d x & \leqq C \int_{0}^{\infty}|f(x)|^{p} w(x) d x, & 1<p<\infty \\
& \leqq C \int_{0}^{\infty}\left(\frac{1}{1+x^{2}}+|f(x)|\left[1+\log ^{+}|f(x)|+\log ^{+}|x|\right]\right) w(x) d x \\
p=1 .
\end{array}
$$

Let $E_{n}=\left[2^{n}, 2^{n+1}\right]$ and $F_{n}=\left[2^{n-1}, 2^{n+2}\right]$. To prove Lemma 7 for $p=1$, use will be made of the fact obtained from Theorem 2, p. 100 of [2] that there is a constant, $C$, such that

$$
\int_{E_{n}}\left|\int_{|x-y|<2^{n-1}} \frac{f(y) d y}{x-y}\right| d x \leqq C \int_{F_{n}}|f(x)|\left(1+\log ^{+} 2^{2 n}|f(x)|\right) d x+C 2^{-n}
$$

$f$ could be assumed to be 0 outside $F_{n}$ in (3.2) since the left side depends only on the values of $f$ in $F_{n}$. Now it is immediate that the absolute value of the difference of $\hat{f}(x)$ and the inner integral on the left side of (3.2) is bounded above by $2^{-n+1} \int_{F_{n}}|f(y)| d y$. From this it follows that there is a $C$ such that

$$
\int_{E_{n}}|\hat{f}(x)| d x \leqq C\left[2^{-n}+\int_{F_{n}}|f(x)|\left(1+\log ^{+} 2^{2 n}|f(x)|\right) d x\right] .
$$

Applying (3.3) to $2^{-2 n} f(x)$ and then multiplying by $2^{2 n}$ gives

$$
\int_{E_{n}}|\hat{f}(x)| d x \leqq C\left[2^{n}+\int_{F_{n}}|f(x)|\left(1+\log ^{+}|f(x)|\right) d x\right] .
$$

Now for each negative integer, $n$, multiply (3.4) for that $n$ by $w\left(2^{n}\right)$ and add these inequalities. For each nonnegative integer, $n$, multiply (3.3) by $w\left(2^{n}\right)$ and add these inequalities. Add these two sums of inequalities together and use the 
assumption about $w(x)$ to take $w\left(2^{n}\right)$ inside the integral signs as $B w(x)$. Then using the fact that $\log ^{+} a b \leqq \log ^{+} a+\log ^{+} b$, gives the result of the lemma for $p=1$.

For $1<p<\infty$, the proof is based on the inequality, $\int_{E_{n}}|\hat{f}(x)|^{p} d x \leqq C \int_{F_{n}}|f(x)|^{p} d x$, easily obtained from Theorem 1, p. 97 of [2]. Lemma 7 is also a special case of Lemma 2 of [3] in this case.

Lemma 8. If $1<p<\infty, r>-1 / p, s<1-1 / p, R<1-1 / p, S>-1 / p, r \geqq R$ and $s \leqq S$, then there exists a constant, $C$, independent of $f$, such that

$$
\int_{-\infty}^{\infty}\left|\int_{-\infty}^{\infty} \frac{f(y)}{x-y} x^{r, s} d y\right|^{p} d x \leqq C \int_{-\infty}^{\infty}\left|f(y) y^{R, s}\right|^{p} d y .
$$

By symmetry it is sufficient to prove this with the outer integral taken over $[0, \infty]$. Now divide the inner integral on the left side of the inequality into integrals over $(-\infty,-x),[-x, 0],\left[0, \frac{1}{2} x\right],\left[\frac{1}{2} x, 3 x / 2\right]$ and $[3 x / 2, \infty)$. In the first of these replace $x-y$ by $-y$ and $f(y)$ by $|f(y)|$, change the variable of integration to $-y$ and use Lemma 4. For the second replace $x-y$ by $x$ and $f(y)$ by $|f(y)|$, change the variable of integration to $-y$ and use Lemma 3. For the third replace $x-y$ by $\frac{1}{2} x$ and $f(y)$ by $|f(y)|$, change the interval of integration to $[0, x]$ and use Lemma 3. For the fourth use Lemma 7 with $w(x)=x^{r, s}$. For the fifth replace $x-y$ by $y / 3$ and $f(y)$ by $|f(y)|$, change the interval of integration to $[x, \infty)$ and use Lemma 4.

Lemma 8 is also a simple consequence of the theorem mentioned at the end of $\S 6$, p. 372 of [8].

LEMmA 9. If $r>-1, s<0, R \leqq 0, S \geqq-1, r \geqq R$ and $s \leqq S$, then there exists a constant, $C$, independent of $f$, such that

$$
\int_{-\infty}^{\infty}\left|\int_{-\infty}^{\infty} \frac{f(y)}{x-y} x^{r, s} d y\right| d x \leqq C+C \int_{-\infty}^{\infty}|f(x)| x^{R, s}\left(1+\log ^{+}|f(x)|+\log ^{+}|x|\right) d x .
$$

The proof is the same as the proof of Lemma 8 with Lemmas 5 and 6 used in place of Lemmas 3 and 4.

Lemma 10. If $1 \leqq p<\infty$, and $T \geqq 1$, there exists a constant, $C$, independent of $T$ and $f(x)$ such that

$$
\begin{aligned}
\int_{0}^{T}\left|\int_{0}^{x} \frac{f(y)(1+x)^{1 / 4}}{x(1+y)^{1 / 4}} d y\right|^{p} d x & \leqq C \theta(p, T) \int_{0}^{T}|f(x)|^{p} d x, & p>1, \\
& \leqq C+C \int_{0}^{T}|f(x)|\left(T^{1 / 4}+\log ^{+}|f(x)|\right) d x, & p=1,
\end{aligned}
$$

where

$$
\begin{array}{rlrl}
\theta(p, T) & =1, & 4 / 3 & <p<\infty \\
& =(\log T)^{4 / 3}, & p & =4 / 3 \\
& =T^{1-3 p / 4}, & 1 \leqq p<4 / 3
\end{array}
$$


If $p>4 / 3$, Lemma 10 is an immediate consequence of Lemma 3 . If $1 \leqq p \leqq 4 / 3$, the integral on the left side of the inequality is bounded above by the sum of

$$
\int_{0}^{1}\left[\int_{0}^{x} \frac{2|f(y)|}{x} d y\right]^{p} d x
$$

and

$$
\int_{1}^{T}\left[\int_{0}^{x} \frac{2|f(y)| x^{-3 / 4}}{(1+y)^{1 / 4}} d y\right]^{p} d x
$$

If $p>1$, Lemma 3 can be applied to (3.5) to give the desired bound and if $p=1$, Lemma 5 can be used. To estimate (3.6), use Hölder's inequality on the inner integral. This shows that (3.6) is bounded by

$$
2^{p} \int_{1}^{T} x^{-3 p / 4}\left(\left[\int_{0}^{x}(1+y)^{-q / 4} d y\right]^{p / q} \int_{0}^{x}|f(y)|^{p} d y\right) d x
$$

where $1 / p+1 / q=1$. Now change the upper limit on the integral of $|f(y)|^{p}$ to $T$. Then by simply computing the integrals, (3.7) can be shown to be bounded by $C \theta(p, T) \int_{0}^{T}|f(y)|^{p} d y$.

LeMmA 11. If $1<p<\infty$, and $T \geqq 1$, there exists a constant, $C$, independent of $T$ and $f(x)$, such that

$$
\int_{-T}^{T}\left|\int_{-T}^{T} \frac{f(y)(1+|x|)^{1 / 4}}{(x-y)(1+|y|)^{1 / 4}} d y\right|^{p} d x \leqq C \theta(p, T) \int_{-T}^{T}|f(x)|^{p} d x .
$$

Lemma 12. If $T \geqq 1$, there exists a constant, $C$, independent of $T$ and $f(x)$, such that

$$
\begin{aligned}
& \int_{-T}^{T}\left|\int_{-T}^{T} \frac{f(y)(1+|x|)^{1 / 4}}{(x-y)(1+|y|)^{1 / 4}} d y\right| d x \\
& \leqq C+C \int_{-T}^{T}|f(x)|\left(T^{1 / 4}+\log ^{+}|f(x)|+\log ^{+}|x|\right) d x .
\end{aligned}
$$

Lemmas 11 and 12 are proved using exactly the same procedure as the proofs of Lemmas 8 and 9 except that Lemma 10 must be used in place of Lemmas 3 or 5 .

LeMmA 13. If $1<p<\infty$, and $T \geqq 1$, there exists a constant, $C$, independent of $T$ and $f(x)$ such that

$$
\int_{-T}^{T}\left|\int_{-T}^{T} \frac{f(y)(1+|y|)^{1 / 4}}{(x-y)(1+|x|)^{1 / 4}} d y\right|^{p} d x \leqq C\left[\theta\left(\frac{p}{p-1}, T\right)\right]^{p-1} \int_{-T}^{T}|f(x)|^{p} d x .
$$

Lemma 13 follows immediately from Lemma 11 by a duality argument.

4. Hermite series theorems. Throughout this section $s_{n}(f, x)$ will denote the $n$th partial sum of the Hermite polynomial series for a function $f(x)$. Theorems 1 and 2 are the general integral inequalities for the partial sums; Theorem 1 includes the result of [1] concerning Hermite series. It will appear in the proof of Theorems 
1 and 2 that it is only the behavior of various terms near $N^{1 / 2}=(2 n+1)^{1 / 2}$ that forces the weight functions $U$ and $V$ to be different for $p$ not in $(4 / 3,4)$. Consequently, the same weight function can be used for both $U$ and $V$ even for $p$ not in $(4 / 3,4)$ provided one of the integrals omits a suitable neighborhood of $N^{1 / 2}$. These results are given in Theorems 3 to 5. Finally, Theorem 6 states the mean convergence results corresponding to Theorems 1 to 5 . Theorem 15 in $\S 8$ gives the proof that the conditions in these theorems cannot be relaxed except possibly in Theorem 1 for the cases when $\beta=1$.

THEOREM 1. Let $1<p<\infty, U(x)=\exp \left(-\frac{1}{2} x^{2}\right)(1+|x|)^{b}$ and

$$
V(x)=\exp \left(-\frac{1}{2} x^{2}\right)(1+|x|)^{B}\left(1+\log ^{+}|x|\right)^{\beta}
$$

where $\beta=1$ if $b=B$ and $p$ is $4 / 3$ or 4 and $\beta=0$ otherwise. Assume that

$$
\begin{aligned}
b & <1-1 / p, & & 1<p \leqq 4, \\
& \leqq 2 / 3+1 / 3 p, & & 4<p<\infty, \\
B & \geqq-1+1 / 3 p, & & 1<p<4 / 3, \\
& >-1 / p, & & 4 / 3 \leqq p<\infty, \\
b & \leqq B+1-4 / 3 p, & & 1<p<4 / 3, \\
& \leqq B, & & 4 / 3 \leqq p \leqq 4, \\
& \leqq B-1 / 3+4 / 3 p, & & 4<p<\infty,
\end{aligned}
$$

and if equality occurs in (4.3), then equality does not occur in (4.1) or (4.2). Then there exists a constant, $C$, independent of $f(x)$ and $n$ such that

$$
\int_{-\infty}^{\infty}\left|s_{n}(f, x) U(x)\right|^{p} d x \leqq C \int_{-\infty}^{\infty}|f(x) V(x)|^{p} d x
$$

TheOREM 2. Let $U(x)=\exp \left(-\frac{1}{2} x^{2}\right)(1+|x|)^{b}$ and $V(x)=\exp \left(-\frac{1}{2} x^{2}\right)(1+|x|)^{B}$ where $b<0, B \geqq-2 / 3$ and $b \leqq B-1 / 3$. Then there exists a constant, $C$, independent of $f(x)$ and $n$ such that

$$
\int_{-\infty}^{\infty}\left|s_{n}(f, x) U(x)\right| d x \leqq C+C \int_{-\infty}^{\infty}|f(x)| V(x)\left(1+\log ^{+}|f(x)|+\log ^{+}|x|\right) d x .
$$

TheOrem 3. Assume that $4 / 3<p<\infty, 0<\delta<1, U(x)=\exp \left(-\frac{1}{2} x^{2}\right)(1+|x|)^{b}$ and $-1 / p<b<1-1 / p$. If $N=2 n+1$ and $O_{n}$ is the set of all $x$ such that ||$x\left|-N^{1 / 2}\right|>\delta N^{1 / 2}$, there exists a constant, $C$, independent of $f$ and $n$ such that

$$
\int_{O_{n}}\left|s_{n}(f, x) U(x)\right|^{p} d x \leqq C \int_{-\infty}^{\infty}|f(x) U(x)|^{p} d x .
$$

TheOREM 4. Assume that $1<p<4, \quad 0<\delta<1, \quad U(x)=\exp \left(\frac{1}{2} x^{2}\right)(1+|x|)^{b}$ and $-1 / p<b<1-1 / p$. If $O_{n}$ is as in Theorem 3 and $f_{n}(x)=f(x)$ on $O_{n}$ and zero off $O_{n}$, 
then there is a constant, $C$, independent of $f$ and $n$ such that

$$
\int_{-\infty}^{\infty}\left|s_{n}\left(f_{n}, x\right) U(x)\right|^{p} d x \leqq C \int_{-\infty}^{\infty}|f(x) U(x)|^{p} d x .
$$

TheOrem 5. Assume that $0<\delta<1, U(x)=\exp \left(-\frac{1}{2} x^{2}\right)(1+|x|)^{b}$ and $-1 \leqq b<0$. If $O_{n}$ is as in Theorem 3 and $f_{n}$ is as in Theorem 4, then there is a constant, $C$, independent of $f$ and $n$, such that

$$
\int_{-\infty}^{\infty}\left|s_{n}\left(f_{n}, x\right) U(x)\right| d x \leqq C+C \int_{-\infty}^{\infty} U(x)|f(x)|\left(1+\log ^{+}|f(x)|+\log ^{+}|x|\right) d x .
$$

THEOREM 6. If the hypotheses of one of the Theorems 1-5 are satisfied and the integral on the right side of its conclusion is finite, then

$$
\lim _{n \rightarrow \infty} \int_{E}\left|\left(s_{n}(x)-f(x)\right) U(x)\right|^{p} d x=0
$$

where $E$ is the set of integration on the left side of the conclusion in the theorem considered, $s_{n}(x)$ is the partial sum considered and $p$ is taken as one in the case of Theorems 2 and 5.

To prove Theorem 1, let $\exp \left(-\frac{1}{2} x^{2}\right) f(x)=g(x), u(x)=(1+|x|)^{b}$ and

$$
v(x)=(1+|x|)^{B}\left(1+\log ^{+}|x|\right)^{\beta} .
$$

Then

$$
s_{n}(f, x)=\exp \left(\frac{1}{2} x^{2}\right) \int_{-\infty}^{\infty} g(y) d_{n}(x, y) d y
$$

where $d_{n}(x, y)$ is the function defined in (2.9). With this notation Theorem 1 is equivalent to showing that there is a $C$ independent of $g$ and $n$ such that

$$
\int_{-\infty}^{\infty}\left|\int_{-\infty}^{\infty} g(y) d_{n}(x, y) u(x) d y\right|^{p} d x \leqq C \int_{-\infty}^{\infty}|g(x) v(x)|^{p} d x
$$

Using (2.16) and symmetry, it is sufficient to show that

$$
\int_{-\infty}^{\infty}\left|\int_{0}^{\infty} g(y) h_{k}(n, x, y) u(x) d y\right|^{p} d x \leqq C \int_{0}^{\infty}|g(x) v(x)|^{p} d x
$$

for $k=1,2$ and 3 .

Proving (4.4) for $k=1$ is easy. Use the definition of $h_{1},(2.11)$, the fact that $\left|\mathscr{H}_{n}(-x)\right|=\left|\mathscr{H}_{n}(x)\right|$ and Hölder's inequality to show that the left side of (4.4) is bounded above by the product of the integral on the right side of (4.4) and

$$
\int_{0}^{\infty}\left|(1+x)^{b} \mathscr{H}_{n}(x)\right|^{p} d x\left(\int_{0}^{\infty}\left|\frac{\mathscr{H}_{n}(x) d y}{(1+y)^{B}\left(1+\log ^{+} y\right)^{\beta}}\right|^{q} d y\right)^{p / q} .
$$

The estimates, (2.3), can now be used to show that (4.5) is a bounded function of $n$. This is simplified if equality does not occur in (4.1) or (4.2) by first observing 
that, if necessary, $b$ can be increased and $B$ decreased so that the hypothesized conditions are still true and $b>-1 / p$ for $1<p \leqq 4, b>-1 / 3+1 / 3 p$ for $4<p$, $B<1 / 3 p$ for $1<p<4 / 3$ and $B<1-1 / p$ for $4 / 3 \leqq p$. With these additional inequalities it is easy to see that the integrals in (4.5) with $\mathscr{H}_{n}(x)$ replaced by the estimates, (2.3), are dominated by the integrals over $\left(\frac{1}{2} N^{1 / 2}, N^{1 / 2}-N^{-1 / 6}\right)$ of the same integrands. It is then easy to prove that (4.5) is bounded for the modified values of $b$ and $B$; this immediately implies that (4.5) is bounded for the original values of $b$ and $B$. If equality occurs in (4.1), $b=2 / 3+1 / 3 p, 4<p<\infty$ and $B>1-1 / p$. If equality occurs in (4.2), $B=-1+1 / 3 p, 1<p<4 / 3$ and $b<-1 / p$. In both these cases it is easy to estimate (4.5) and show that it is bounded.

To prove (4.4) for $k=2$ and 3 , recall the notation $N=2 n+1$, and write $g(x)$ $=g_{1}(x)+g_{2}(x)+g_{3}(x)$ where the $g_{j}$ 's equal $g$ on $\left[0, \frac{1}{2} N^{1 / 2}\right],\left[\frac{1}{2} N^{1 / 2}, 3 N^{1 / 2} / 2\right]$ and $\left[3 N^{1 / 2} / 2, \infty\right)$ respectively and are 0 elsewhere. Let $E_{1}=\left[-\frac{1}{4} N^{1 / 2}, 3 N^{1 / 2} / 4\right], E_{2}$ $=\left[\frac{1}{4} N^{1 / 2}, 7 N^{1 / 2} / 4\right]$ and $E_{3}=\left[5 N^{1 / 2} / 4, \infty\right)$ and $\widetilde{E}_{j}$ denote the complement of $E_{j}$ in $(-\infty, \infty)$. Then for $k=2$ and 3 the left side of (4.4) is bounded above by a constant times the sum of

$$
\sum_{j=1}^{3} \int_{\tilde{E}_{j}}\left|\int_{0}^{\infty} g_{j}(y) h_{k}(n, x, y)(1+|x|)^{b} d y\right|^{p} d x
$$

and

$$
\sum_{j=1}^{3} \int_{E_{j}}\left|\int_{0}^{\infty} g_{j}(y) h_{k}(n, x, y)(1+|x|)^{b} d y\right|^{p} d x
$$

Because of the definitions of $E_{j}$ and $g_{j}$, the integration in (4.6) is confined to points, $(x, y)$, such that $|x-y| \geqq \frac{1}{4} N^{1 / 2}$. Consequently, in (4.6) $\left|h_{2}(n, x, y)\right|$ $\leqq 2\left|\mathscr{H}_{n}(y)\right|\left|\mathscr{H}_{n+1}(x)-\mathscr{H}_{n-1}(x)\right|$ and $\left|h_{3}\right|$ is bounded by the same expression with the $x$ and $y$ reversed. Then the estimates, (2.3), can be applied and the computation used to estimate (4.4) for $k=1$ can be repeated here.

This leaves only the consideration of (4.7) for $k=2$ and $k=3$. In the individual integrals of (4.7) with $j=1$ and $k=2$ or 3 use the estimates (2.3) and (2.4) to show that these double integrals are equal to

$$
\int_{E_{1}}\left|\int_{E_{1}} \frac{g_{1}(y) \phi(x, n) \psi(y, n)(1+|x|)^{b}}{x-y} d y\right|^{p} d x
$$

here and hereafter $\phi$ and $\psi$ will be used to denote functions uniformly bounded in both variables but not necessarily the same at each occurrence. Then Lemma 8 can be applied to (4.8) to give the desired estimate since $b<1-1 / p, b \leqq B$ and $B>-1 / p$.

Using (2.3) and (2.4), the integral in (4.7) with $j=2$ and $k=2$ becomes

$$
\int_{E_{2}}\left|\int_{E_{2}} \frac{g_{2}(y)\left(\frac{1}{2} N^{-1 / 6}+\left|x-N^{1 / 2}\right|\right)^{1 / 4} \phi(x, n) \psi(y, n) N^{b / 2}}{(x-y)\left(\frac{1}{2} N^{-1 / 6}+\left|y-N^{1 / 2}\right|\right)^{1 / 4}} d y\right|^{p} d x
$$


Now make the changes of variable $x=N^{1 / 2}+\frac{1}{2} N^{-1 / 6} X$ and $y=N^{1 / 2}+\frac{1}{2} N^{-1 / 6} Y$; this changes (4.9) into

$$
\int_{-N^{2 / 3}}^{N^{2 / 3}}\left|\int_{-N^{2 / 3}}^{N^{2 / 3}} \frac{g_{2}(y)}{X-Y} \frac{(1+X)^{1 / 4}}{(1+Y)^{1 / 4}} \phi(X, n) \psi(Y, n) N^{B / 2} d Y\right|^{p} N^{-1 / 6} d X .
$$

By Lemma 11, (4.10) is bounded by

$$
C \theta\left(p, N^{2 / 3}\right) \int_{-N^{2 / 3}}^{N^{2 / 3}}\left|N^{B / 2} g_{2}(x)\right|^{p} N^{-1 / 6} d X .
$$

Then changing the variable back to $x,(4.11)$ becomes

$$
C \theta\left(p, N^{2 / 3}\right) N^{B p / 2} \int_{E_{2}}\left|g_{2}(x)\right|^{p} d x .
$$

Because of the definition of $E_{2}$, the power of $N$ can be taken inside the integral in (4.12) as a power of $1+|x|$ and a power of $\log N$ can be taken inside as a power of $1+\log ^{+}|x|$. This will give the desired bound.

The estimation of the integral in (4.7) with $j=2$ and $k=3$ is the same except that Lemma 13 is used in place of Lemma 11.

For the integral in (4.7) with $j=3$ and $k=2$ or 3 , the estimates (2.3) and (2.4) show that this integral is bounded above by

$$
\int_{E_{3}}\left|\int_{E_{3}} \frac{f(y)}{x-y}(1+x)^{b} \phi(x, n) \psi(y, n) d y\right|^{p} d x
$$

since $N^{1 / 2} \exp (-\gamma N)$ is a bounded function of $N$. Lemma 8 can then be applied. This completes the proof that (4.7) is bounded by the right side of (4.4) for $k=2$ and $k=3$ and thereby completes the proof of Theorem 1 .

Theorem 2 is proved in the same way with Lemma 9 used in place of Lemmas 8 and 13 and Lemma 12 in place of Lemma 11. One new feature occurs if $B=-2 / 3$ and $b=-1$ since (4.5) is not bounded; in this case it is easy to estimate directly the left side of (4.4) for $k=1$.

The proof of Theorems 3 and 4 are like that of Theorem 1. For these proofs $g_{1}, g_{2}$ and $g_{3}$ should be taken equal to $g$ on $\left[0,\left(1-\frac{1}{2} \delta\right) N^{1 / 2}\right],\left[\left(1-\frac{1}{2} \delta\right) N^{1 / 2},\left(1+\frac{1}{2} \delta\right) N^{1 / 2}\right]$ and $\left[\left(1+\frac{1}{2} \delta\right) N^{1 / 2}, \infty\right)$ respectively and

and

$$
\begin{aligned}
& E_{1}=\left[-\frac{1}{4} N^{1 / 2},\left(1-\frac{1}{4} \delta\right) N^{1 / 2}\right], \\
& E_{2}=\left[(1-\delta) N^{1 / 2},(1+\delta) N^{1 / 2}\right]
\end{aligned}
$$

$$
E_{3}=\left[\left(1+\frac{1}{4} \delta\right) N^{1 / 2}, \infty\right)
$$

The computations are simpler for these theorems and in the analogue of (4.7) the term for $j=2$ drops out. The proof of Theorem 5 is based on that of Theorem 2 with the modifications just described. 
Theorem 6 is proved in the standard way. Consider the part concerning Theorem 1 and let \|\|$_{p}$ denote the unweighted $L^{p}$ norm on $(-\infty, \infty)$. Given $\varepsilon>0$ and $f(x)$ a function such that $f(x) V(x) \in L^{p}$, let $C$ be the constant in Theorem 1. Then by Lemma 2 there is a polynomial, $g(x)$, such that

$$
\|(f(x)-g(x)) V(x)\|_{p}<\varepsilon /(1+C) .
$$

Since $U(x) \leqq V(x)$, then

$$
\|(f(x)-g(x)) U(x)\|_{p}<\varepsilon /(1+C) .
$$

Using Theorem 1 and (4.13) shows that

$$
\left\|\left(s_{n}(f, x)-s_{n}(g, x)\right) U(x)\right\|_{p} \leqq C\|(f(x)-g(x)) V(x)\|_{p}<C \varepsilon /(1+C) .
$$

If $M$ is the degree of $g$, then for $n>M s_{n}(g, x)=g(x)$. Combining this fact, (4.14), (4.15) and Minkowski's inequality shows that if $n>M$, then $\left\|\left(f(x)-s_{n}(f, x)\right) U(x)\right\|_{p}$ $<\varepsilon$. This proves Theorem 6 as it concerns Theorem 1 .

The proof of Theorem 6 as it applies to Theorem 3 is exactly the same as the proof above.

The proof of Theorem 6 as it concerns Theorem 4 must be slightly different since $s_{n}\left(g_{n}, x\right)$ will never equal $g(x)$ if $g$ is a polynomial. For this proof let $U(x)$ and $C$ be as in Theorem 4. Given $\varepsilon>0$ and $f(x)$ such that $f(x) U(x) \in L^{p}$, let $g(x)$ be a bounded function with compact support that satisfies (4.14). Since for the $U(x)$ in Theorem $4 b<1-1 / p$ and $1<p<4$, there is a $V(x)$ such that $U(x)$ and $V(x)$ are a pair of weight functions satisfying the hypotheses of Theorem 1. Since $g$ is bounded and has compact support, $g(x) V(x) \in L^{p}$. Then using the application of Theorem 6 to Theorem 1 shows that $\lim _{n \rightarrow \infty}\left\|\left(s_{n}(g, x)-g(x)\right) U(x)\right\|_{p}=0$. Furthermore, for $n$ sufficiently large $s_{n}\left(g_{n}, x\right)=s_{n}(g, x)$. These facts can then be combined as before to prove that Theorem 6 applies to Theorem 4 .

The proof of the part of Theorem 6 concerning Theorem 2 is complicated by the involved expression on the right side of the conclusion of Theorem 2. For this proof let $C$ be the larger of 1 and the constant in Theorem 2 and $f$ be a function for which $\left\|V(x) f(x)\left[1+\log ^{+}|x|+\log ^{+}|f(x)|\right]\right\|_{1}$ is finite. Given $\varepsilon>0$, let $g(x)$ be a bounded function with compact support such that

$$
\frac{\varepsilon}{8}+C\left\|(f(x)-g(x))\left(1+\log ^{+}|x|+\log ^{+} \frac{8 C|f(x)-g(x)|}{\varepsilon}\right) V(x)\right\|_{1}
$$

is less than $\frac{1}{4} \varepsilon$. Applying Theorem 2 to $8 C(f(x)-g(x)) / \varepsilon$ and then multiplying by $\varepsilon / 8 C$ shows that $\left\|s_{n}(f-g, x) U(x)\right\|_{1}$ is bounded above by (4.16). Therefore

$$
\left\|\left(s_{n}(f, x)-s_{n}(g, x)\right) U(x)\right\|_{1}<\frac{1}{4} \varepsilon .
$$

Since $U(x) \leqq V(x)$, it is also true that

$$
\|(f(x)-g(x)) U(x)\|_{1}<\frac{1}{4} \varepsilon .
$$


By Lemma 2 there is a polynomial, $h(x)$, such that

$$
\varepsilon / 8+C S^{1 / 2}\left(\|(1+|x|) V(x)\|_{1}\right)^{1 / 2}+8 C^{2} S / \varepsilon
$$

is less than $\frac{1}{4} \varepsilon$ where $S=\left\|(g(x)-h(x))^{2}(1+|x|) V(x)\right\|_{1}$. Schwarz's inequality shows that (4.19) is an upper bound for

$$
\varepsilon / 8+C\|(g(x)-h(x))(1+|x|) V(x)\|_{1}+\left(8 C^{2} / \varepsilon\right)\left\|(g(x)-h(x))^{2} V(x)\right\|_{1} .
$$

Using the fact $\log ^{+}|z| \leqq|z|$ shows that (4.20) is an upper bound for expression (4.16) with $f$ replaced by $h$. Therefore,

$$
\left\|\left(s_{n}(g, x)-s_{n}(h, x)\right) U(x)\right\|_{1}<\frac{1}{4} \varepsilon
$$

and

$$
\|(g(x)-h(x)) U(x)\|_{1}<\frac{1}{4} \varepsilon .
$$

Since $s_{n}(h, x)=h(x)$ for $n$ sufficiently large, (4.17), (4.18), (4.21) and (4.22) can be combined to show that $\left\|\left(s_{n}(f, x)-f(x)\right) U(x)\right\|_{1}<\varepsilon$ for $n$ sufficiently large.

The proof that Theorem 6 applies to Theorem 5 is like the proof that Theorem 6 applies to Theorem 4. Use is made of some parts of the proof that Theorem 6 applies to Theorem 2 .

5. Laguerre series theorems. Throughout this section $s_{n}^{\alpha}(f, x)$ will denote the $n$th partial sum of the Laguerre polynomial series for $f(x)$ with parameter $\alpha$. The theorems stated here are the exact analogues of the Hermite series theorems but are somewhat more complicated since the weight functions now contain powers of $x /(1+x)$. Again, the proof that the conditions cannot be significantly relaxed is in Theorem 15 in $\S 8$.

THEOREM 7. Let $1<p<\infty, \quad \alpha>-1, \quad U(x)=e^{-x / 2} x^{\alpha / 2}(x /(1+x))^{a}(1+x)^{b}$ and $V(x)=e^{-x / 2} x^{\alpha / 2}(x /(1+x))^{A}(1+x)^{B}\left(1+\log ^{+} x\right)^{\beta}$ where $\beta=1$ if $b=B$ and $p$ is $4 / 3$ or 4 and $\beta=0$ otherwise. Assume that

$$
a>-1 / p+\max \left(-\frac{1}{2} \alpha, \frac{1}{4}\right), \quad A<1-1 / p-\max \left(-\frac{1}{2} \alpha, \frac{1}{4}\right), \quad A \leqq a,
$$

$$
\begin{array}{rlrl}
b & <3 / 4-1 / p, & 1 & <p \leqq 4, \\
b & \leqq 7 / 12-1 / 3 p, & 4 & <p<\infty, \\
B & \geqq-\frac{1}{4}-1 / 3 p, & 1 & <p<4 / 3, \\
& >\frac{1}{4}-1 / p, & 4 / 3 \leqq p & <\infty, \\
b & \leqq B+\frac{1}{2}-2 / 3 p, & 1 & <p<4 / 3, \\
& \leqq B, & 4 / 3 \leqq p \leqq 4, \\
& \leqq B-1 / 6+2 / 3 p, & 4 & <p<\infty,
\end{array}
$$


and if equality occurs in (5.3), then equality does not occur in (5.1) or (5.2). Then there exists a constant, $C$, independent of $f(x)$ and $n$, such that

$$
\int_{0}^{\infty}\left|s_{n}^{\alpha}(f, x) U(x)\right|^{p} d x \leqq C \int_{0}^{\infty}|f(x) V(x)|^{p} d x .
$$

THEOREM 8. Let $\alpha>-1$, and let $U(x)$ and $V(x)$ have the form used in Theorem 7 with $\beta=0$. Assume that $a \geqq A, a>-1+\max \left(-\frac{1}{2} \alpha, \frac{1}{4}\right), A \leqq \min \left(\frac{1}{2} \alpha,-\frac{1}{4}\right), b<-\frac{1}{4}$, $B \geqq-7 / 12$ and $b \leqq B-1 / 6$. Then there is a constant, $C$, independent of $f$ and $n$, such that

$$
\int_{0}^{\infty}\left|s_{n}^{\alpha}(f, x) U(x)\right| d x \leqq C+C \int_{0}^{\infty}|f(x)| V(x)\left(1+\log ^{+}|f(x)|+\log ^{+} x\right) d x .
$$

TheOREM 9. Assume that $\alpha>-1,4 / 3<p<\infty$ and $0<\delta<1$. Let

$$
U(x)=e^{-x / 2} x^{\alpha / 2}(x /(1+x))^{a}(1+x)^{b}
$$

and for a given $n$ let $\nu=4 n+2 \alpha+2$ and $O_{n}$ be the set of all $x$ such that $x>0$ and $|x-\nu|>\delta \nu$. If $-1 / p+\max \left(-\frac{1}{2} \alpha, \frac{1}{4}\right)<a<1-1 / p-\max \left(-\frac{1}{2} \alpha, \frac{1}{4}\right)$ and $-1 / p+\frac{1}{4}<b$ $<-1 / p+\frac{3}{4}$, then there exists a constant, $C$, independent of $f$ and $n$, such that

$$
\int_{O_{n}}\left|s_{n}^{\alpha}(f, x) U(x)\right|^{p} d x \leqq C \int_{0}^{\infty}|f(x) U(x)|^{p} d x .
$$

THEOREM 10. Assume that $\alpha>-1,1<p<4,0<\delta<1$ and $U(x)$ and $O_{n}$ are as in Theorem 9 with the same conditions on $a$ and $b$. Let $f_{n}(x)=f(x)$ on $O_{n}$ and be 0 off $O_{n}$. Then there exists a constant, $C$, independent of $f$ and $n$, such that

$$
\int_{0}^{\infty}\left|s_{n}^{\alpha}\left(f_{n}, x\right) U(x)\right|^{p} d x \leqq C \int_{0}^{\infty}|f(x) U(x)|^{p} d x .
$$

Theorem 11. Assume that $\alpha>-1,0<\delta<1, U(x)$ and $O_{n}$ are as in Theorem 9, $-1+\max \left(-\frac{1}{2} \alpha, \frac{1}{4}\right)<a \leqq \min \left(\frac{1}{2} \alpha,-\frac{1}{4}\right)$ and $-\frac{3}{4} \leqq b<-\frac{1}{4}$. Then there exists $a$ constant, $C$, independent of $f$ and $n$, such that

$$
\int_{0}^{\infty}\left|s_{n}^{\alpha}\left(f_{n}, x\right) U(x)\right| d x \leqq C+C \int_{0}^{\infty}|f(x)| U(x)\left(1+\log ^{+}|f(x)|+\log ^{+} x\right) d x .
$$

THEOREM 12. If the hypotheses of one of the Theorems 7-11 are satisfied and the integral on the right side of its conclusion is finite, then

$$
\lim _{n \rightarrow \infty} \int_{E}\left[\left|s_{n}^{\alpha}(x)-f(x)\right| U(x)\right]^{p} d x=0
$$

where $E$ is the set of integration on the left side of the conclusion in the theorem considered, $s_{n}(x)$ is the partial sum considered and $p$ is taken as one in the case of Theorems 8 and 11 .

The proof of Theorem 7 is similar to that of Theorem 1 but with a few additional complications. For this proof let $f(x)=e^{x / 2} x^{-\alpha / 2} g(x), U(x)=e^{-x / 2} x^{\alpha / 2} u(x)$ and 
$V(x)=e^{-x / 2} x^{\alpha / 2} v(x)$. Then $s_{n}^{\alpha}(f, x)=e^{x / 2} x^{-\alpha / 2} \int_{0}^{\infty} g(y) D_{n}(x, y) d y$ where $D_{n}(x, y)$ is the function defined in (2.17). Using this in the conclusion of Theorem 7 and, using (2.22) shows that it is sufficient to prove that

$$
\int_{0}^{\infty}\left|\int_{0}^{\infty} g(y) j_{1}(n, x, y) u(x) d y\right|^{p} d x=C \int_{0}^{\infty}|g(x) v(x)|^{p} d x
$$

and

$$
\int_{0}^{\infty}\left|\int_{0}^{\infty} g(y)\left(j_{2}(n, x, y)+j_{3}(n, x, y)\right) u(x) d y\right|^{p} d x \leqq C \int_{0}^{\infty}|g(x) v(x)|^{p} d x
$$

As in the Hermite case, the proof of (5.4) is easy since it reduces to showing that

$$
\int_{0}^{\infty}\left|\mathscr{L}_{n}^{\alpha}(x) u(x)\right|^{p} d x\left[\int_{0}^{\infty}\left|\mathscr{L}_{n}^{\alpha}(y) / v(y)\right|^{q} d y\right]^{p / q}
$$

is a bounded function of $n$ where $1 / p+1 / q=1$. Now if equality does not hold in (5.1) or (5.2), $b$ can be increased and $B$ decreased so that the original hypotheses are still true and $b>\frac{1}{4}-1 / p$ for $1<p \leqq 4, b>1 / 12-1 / 3 p$ for $4<p<\infty, B<\frac{1}{4}-1 / 3 p$ for $1<p<4 / 3$ and $B<3 / 4-1 / p$ for $4 / 3 \leqq p<\infty$. Using these additional inequalities and the estimates, (2.5), it is clear that the integrals in (5.6) with $\mathscr{L}_{n}^{\alpha}$ replaced by its estimate are dominated by the integrals over $\left(\frac{1}{2} \nu, \nu-\nu^{1 / 3}\right)$ of the same integrands. It is then easy to show that (5.6) is bounded. If equality occurs in (5.1) or (5.2), it is also easy to prove directly that (5.6) is bounded.

To prove (5.5) recall that $\nu=4 n+2 \alpha+2$ and write $g(x)=\sum_{i=1}^{4} g_{i}(x)$ where the $g_{i}$ 's equal $g$ on $[0,1 / \nu],\left[1 / \nu, \frac{1}{2} \nu\right],\left[\frac{1}{2} \nu, 3 \nu / 2\right]$ and $[3 \nu / 2, \infty)$ respectively and are 0 elsewhere. Let $E_{1}=[0,2 / \nu], E_{2}=[1 / 2 v, 3 v / 4], E_{3}=\left[\frac{1}{4} v, 7 v / 4\right]$, and $E_{4}=[5 v / 4, \infty)$ and let $\widetilde{E}_{i}$ denote the complement of $E_{i}$ in $[0, \infty)$. Then the left side of $(5.5)$ is bounded by a constant times the sum of

$$
\sum_{i=1}^{4} \int_{\tilde{E}_{i}}\left[\int_{0}^{\infty}\left|g_{i}(y)\left(j_{2}(n, x, y)+j_{3}(n, x, y)\right)\right| u(x) d y\right]^{p} d x
$$

and

$$
\sum_{i=1}^{4} \int_{E_{i}}\left|\int_{0}^{\infty} g_{i}(y)\left(j_{2}(n, x, y)+j_{3}(n, x, y)\right) u(x) d y\right|^{p} d x
$$

In (5.7), if the outer integration is confined to $\left[\frac{1}{2} \nu, \infty\right)$ in the case $i=1,(3 \nu / 4, \infty)$ in the case $i=2$ and is taken to be all of $\widetilde{E}_{i}$ for $i=3$ or 4 , then the integrations are taken over pairs, $(x, y)$, such that $|x-y| \geqq \frac{1}{4} \nu$. With this condition, $\left|j_{2}(n, x, y)\right|$ $\leqq 4\left|\mathscr{L}_{n}^{\alpha}(y)\left(\mathscr{L}_{n+1}^{\alpha}(x)-\mathscr{L}_{n-1}^{\alpha}(x)\right)\right|$ and the computation used to deal with $j_{1}$ can be repeated. The same applies to $j_{3}$. It follows that these parts of (5.7) have the desired bound. The remaining part of (5.7) is the sum of

$$
\int_{2 / v}^{v / 2}\left[\int_{0}^{1 / v}\left|g(y)\left(j_{2}(n, x, y)+j_{3}(n, x, y)\right)\right| u(x) d y\right]^{p} d x
$$


and

$$
\int_{0}^{1 / 2 v}\left[\int_{1 / v}^{v / 2}\left|g(y)\left(j_{2}(n, x, y)+j_{3}(n, x, y)\right)\right| u(x) d y\right]^{p} d x
$$

Substitute the values of $j_{2}$ and $j_{3}$ in (5.9) and (5.10) and replace the $\mathscr{L}_{n}^{\alpha}$ 's by the estimates (2.5) and (2.6). The estimate for the $j_{3}$ part of (5.9) is less than the estimate for the $j_{2}$ part; similarly the estimate for the $j_{2}$ part of (5.10) is bounded by the estimate for the $j_{3}$ part. This leaves the following two integrals to estimate:

$$
\int_{2 / v}^{v / 2}\left[\int_{0}^{1 / v} \nu^{\alpha / 2+1 / 4}|g(y)| y^{\alpha / 2} x^{-3 / 4} u(x) d y\right]^{p} d x
$$

and

$$
\int_{0}^{1 / 2 v}\left[\int_{1 / v}^{v / 2} \nu^{\alpha / 2+1 / 4}|g(y)| y^{-3 / 4} x^{\alpha / 2} u(x) d y\right]^{p} d x .
$$

These are easy to estimate by integrating with respect to $x$ first and using Hölder's inequality on the other part in such a way that one of the integrals has integrand $|g(y) v(y)|^{p}$. This estimation completes the consideration of (5.7).

To estimate the part of (5.8) with $i=1$, observe that (2.5) implies that given an $n,\left|\mathscr{L}_{k}^{\alpha}(x)\right| \leqq C x^{\alpha / 2} \nu^{\alpha / 2}$ for $0 \leqq k \leqq n$ and $0 \leqq x \leqq 2 / \nu$. This and the definition, (2.17), of $D_{n}$ implies that for $x$ and $y$ in $E_{1}$

$$
\left|D_{n}(x, y)\right| \leqq C x^{\alpha / 2} y^{\alpha / 2} \nu^{\alpha+1} .
$$

Since $j_{1}(n, x, y)$ is also bounded above by this for $x$ and $y$ in $E_{1},(2.22)$ implies that $\left|j_{2}+j_{3}\right|$ is also bounded above by the right side of (5.13). Using this on the part of (5.8) with $i=1$ produces the integral

$$
C \int_{0}^{2 / v}\left|\int_{0}^{2 / v} \nu^{\alpha+1} g_{1}(y) y^{\alpha / 2} x^{\alpha / 2+a} d y\right|^{p} d x .
$$

Hölder's inequality and the hypotheses of Theorem 7 then show easily that (5.14) is bounded above by the right side of (5.5).

For the other parts of (5.10) replace $j_{2}$ and $j_{3}$ by their values and use the estimates (2.5) and (2.6). After suppressing bounded functions that depend only on $x$ and $n$ and bounded functions that depend only on $y$ and $n$, there remain the following integrals :

$$
\begin{gathered}
\int_{E_{2}}\left|\int_{E_{2}} \frac{g_{2}(y)}{x-y}\left(\frac{y}{x}\right)^{ \pm 1 / 4}\left(\frac{x}{1+x}\right)^{a}(1+x)^{b} d y\right|^{p} d x, \\
\int_{E_{3}}\left|\int_{E_{3}} \frac{g_{3}(y)}{x-y}\left(\frac{\nu^{1 / 3}+|v-x|}{\nu^{1 / 3}+|\nu-y|}\right)^{ \pm 1 / 4} n^{b} d y\right|^{p} d x
\end{gathered}
$$

and

$$
\int_{E_{4}}\left|\int_{E_{4}} \frac{g_{4}(y)}{x-y}(1+x)^{b} d y\right|^{p} d x
$$


Each of these must be shown to be bounded by the right side of (5.5). For (5.15) and (5.17) this follows immediately from the conditions on $a, A, b$ and $B$ and Lemma 8. For (5.16) make the change of variable $X=\nu^{-1 / 3}(\nu-x), Y=\nu^{-1 / 3}(\nu-y)$ and proceed by using Lemmas 11 and 13 as was done in the estimation of (4.9).

This completes the proof of Theorem 7 .

Theorems 8 through 11 are proved by modifying the proof of Theorem 7 in the same way that Theorems 2 through 5 were proved by modifying the proof of Theorem 1. Theorem 12 is proved in the same way as Theorem 6 . There are a few additional complications that appear but they are easy to treat.

6. Justifying the weight functions. This section and the next two sections are devoted to showing that in various ways Theorems 1 through 12 are the best obtainable. The first problem is to show that the weight functions used are the only reasonable ones that could be used. This is done as it was sketched in $\S 1$ by use of Theorems 13 and 14.

THEOREM 13. Assume that $p$ is a fixed number such that $1<p<\infty$, define $q$ by $1 / p+1 / q=1$ and let $s_{n}(f, x)$ be the nth partial sum of $f$ 's Hermite polynomial series. Assume that $U(x)$ and $V(x)$ are functions such that $U(x)$ is not zero on a set of positive measure, $V(x)$ is finite a.e. and

$$
\int_{-\infty}^{\infty}\left|s_{n}(f, x) U(x)\right|^{p} d x \leqq K \int_{-\infty}^{\infty}|f(x) V(x)|^{p} d x
$$

where $K$ is a constant independent of $f$ and $n$. Then there is a constant, $C$, such that for every $t \geqq 1$

$$
\int_{-t}^{t}\left|U(x) \exp \left(\frac{1}{2} x^{2}\right)\right|^{p} d x \leqq C t^{p}
$$

and

$$
\int_{-t}^{t}\left|V(x) \exp \left(\frac{1}{2} x^{2}\right)\right|^{-q} d x \leqq C t^{q}
$$

THEOREM 14. Assume that $\alpha>-1$ and $p$ is a fixed number such that $1<p<\infty$, define $q$ by $1 / p+1 / q=1$ and let $s_{n}(f, x)$ denote the nth partial sum of $f$ 's Laguerre polynomial series for this $\alpha$. Assume that $U(x)$ and $V(x)$ are functions such that $U(x)$ is not zero on a subset of $(0, \infty)$ of positive measure, $V(x)$ is finite a.e. and

$$
\int_{-\infty}^{\infty}\left|s_{n}(f, x) U(x)\right|^{p} d x \leqq K \int_{-\infty}^{\infty}|f(x) V(x)|^{p} d x
$$

where $K$ is a constant independent of $f$ and $n$. Then there is a constant, $C$, such that for every $t \geqq 1$

$$
\int_{1 / t}^{t}\left|U(x) x^{-1 / 4-\alpha / 2} e^{x / 2}\right|^{p} d x \leqq C t^{p / 2}
$$

and

$$
\int_{1 / t}^{t}\left|V(x) x^{1 / 4-\alpha / 2} e^{x / 2}\right|^{-q} d x \leqq C t^{q / 2}
$$


The proofs are fairly simple consequences of various theorems in [4]. To prove Theorem 14 let $u(x)=U(x) x^{-\alpha / 2} e^{x / 2}$ and $v(x)=V(x) x^{-\alpha / 2} e^{x / 2}$. The obvious modification of Lemma 1 of [4] to take account of the two weight functions shows that there is a constant $C$ such that

$$
\left\|\mathscr{L}_{n}^{\alpha}(x) u(x)\right\|_{p}\left\|\mathscr{L}_{n}^{\alpha}(x) / v(x)\right\|_{q} \leqq C
$$

where \|\|$_{p}$ denotes the unweighted norm on $(0, \infty)$. Lemma 13 of [4] shows that each factor in (6.5) is bounded below by a constant times $n^{-1 / 4}$. Consequently, there is a constant, $C$, such that if $n \geqq 1$, then

$$
\left\|\mathscr{L}_{n}^{\alpha}(x) u(x)\right\|_{p} \leqq C n^{1 / 4}
$$

and

$$
\left\|\mathscr{L}_{n}^{\alpha}(x) / v(x)\right\|_{q} \leqq C n^{1 / 4} .
$$

By Lemma 7 of [4] there are constants $s_{0}$ and $C>0$ such that if $s \geqq s_{0}$, then there is an $n$ satisfying $s / 5 \leqq n \leqq s / 3$ and

$$
\int_{E_{s}}\left|\mathscr{L}_{n}^{\alpha}(x) u(x)\right|^{p} d x \geqq C \int_{E_{s}}\left|s^{-1 / 2} u(x)\right|^{p} d x
$$

where $E_{s}=[5 s / 6,11 s / 12]$. Combining (6.8) and (6.6) then leads to

$$
\int_{E_{s}}\left|x^{-1 / 4} u(x)\right|^{p} d x \leqq C s^{p / 2}
$$

for all $s \geqq s_{0}$.

Now, (6.3) will be proved by showing that

$$
\int_{1}^{t}\left|x^{-1 / 4} u(x)\right|^{p} d x \leqq C t^{p / 2}
$$

and

$$
\int_{1 / t}^{1}\left|x^{-1 / 4} u(x)\right|^{p} d x \leqq C t^{p / 2}
$$

Since (6.6) is true for all $n \geqq 1,|u(x)|^{p}$ is integrable on [1, $\left.s_{0}\right]$, and by adjusting $C(6.10)$ is true for $1 \leqq t \leqq s_{0}$. Then (6.10) can be obtained for all $t \geqq 1$ by adding (6.10) for $t=s_{0}$ to inequalities of the type (6.9) for appropriate values of $s$.

To prove (6.11), use can be made of the fact from [5] (5.1.6), p. 100 that

$$
L_{n}^{\alpha}(x)=\sum_{k=0}^{n}\left(\begin{array}{l}
n+\alpha \\
n-k
\end{array}\right) \frac{(-x)^{k}}{k !} .
$$

If $0 \leqq x \leqq(1+\alpha) / 3 n$, the constant term is greater than twice the sum of the other terms and

$$
L_{n}^{\alpha}(x) \geqq \frac{1}{2}\left(\begin{array}{c}
n+\alpha \\
n
\end{array}\right)
$$


Therefore, there is a positive constant, $C$, depending only on $\alpha$ such that for $n \geqq 1$

$$
\mathscr{L}_{n}^{\alpha}(x) \geqq C(n x)^{\alpha / 2}, \quad 0 \leqq x \leqq \frac{1+\alpha}{3 n}
$$

Then restricting the integration in (6.6) to $G_{n}=[(1+\alpha) / 6 n,(1+\alpha) / 3 n]$ and using (6.12) shows that for $n \geqq 1$

$$
\int_{G_{n}}\left|x^{-1 / 4} u(x)\right|^{p} d x \leqq C n^{p / 2} .
$$

Therefore, there is an $s_{0}$ and $C$ such that if $s \geqq s_{0}$, then

$$
\int_{1 / s}^{2 / s}\left|x^{-1 / 4} u(x)\right|^{p} d x \leqq C s^{p / 2}
$$

As in the proof of (6.10), the $C$ in (6.11) can be adjusted to make (6.11) true for $1 \leqq t \leqq s_{0}$. Then adding in the inequalities (6.14) for appropriate values of $s$ will prove (6.11) in general.

The inequality (6.4) follows from (6.7) by exactly the same reasoning.

Theorem 13 is proved in the same way that (6.10) was proved using Lemmas 12 and 6 of [4].

7. Estimating integrals. Lower bounds for various integrals will be needed to prove Theorem 15 in $\S 8$. To obtain these, some expressions for $\mathscr{H}_{n}(x)$ and $\mathscr{L}_{n}^{\alpha}(x)$ are given. Then two lemmas about these expressions, Lemmas 14 and 15 are proved. Lemma 15 leads to the needed integral estimates.

Basic to the expressions for $\mathscr{H}_{n}(x)$ and $\mathscr{L}_{n}^{\alpha}(x)$ will be the function

$$
g(N, x)=-\frac{1}{2} N \cos ^{-1}\left(x N^{-1 / 2}\right)+\frac{1}{2} x\left(N-x^{2}\right)^{1 / 2}+\frac{1}{4} \pi .
$$

As usual, the notation $N=2 n+1$ and $\nu=4 n+2 \alpha+2$ will be used. The arguments for $g$ will be assumed to be $(N, x)$ in the Hermite expressions and $\left(\nu, x^{1 / 2}\right)$ in the Laguerre expressions. The following will be needed:

$$
\begin{aligned}
& \mathscr{H}_{n}(x)=\frac{(2 / \pi)^{1 / 2}}{\left(N-x^{2}\right)^{1 / 4}} \cos g+O\left(\frac{N^{1 / 2}}{\left(N-x^{2}\right)^{7 / 4}}\right), \quad 1 \leqq x \leqq N^{1 / 2}-N^{-1 / 6} \\
& \mathscr{H}_{n+1}(x)-\mathscr{H}_{n-1}(x)=\frac{2^{3 / 2}}{\pi^{1 / 2} N^{1 / 4}} \sin g+O\left(x N^{-3 / 4}\right), \quad 1 \leqq x \leqq \frac{1}{2} N^{1 / 2} \\
& \mathscr{L}_{n}^{\alpha}(x)=\frac{(2 / \pi)^{1 / 2}}{(\nu x)^{1 / 4}}\left(\cos \left[(\nu x)^{1 / 2}-\frac{\alpha \pi}{2}-\frac{\pi}{4}\right]+O\left(\nu^{-1 / 2} x^{-1 / 2}\right)\right), \quad \frac{1}{\nu} x \leqq 1, \\
& \mathscr{L}_{n+1}^{\alpha}(x)-\mathscr{L}_{n-1}^{\alpha}(x)=\frac{-2^{5 / 2} x^{1 / 4}}{\pi^{1 / 2} \nu^{3 / 4}}\left(\sin \left[(\nu x)^{1 / 2}-\frac{\alpha \pi}{2}-\frac{\pi}{4}\right]+O\left[x+(\nu x)^{-1 / 2}\right]\right), \\
& \mathscr{L}_{n}^{\alpha}(x)=\frac{(2 / \pi)^{1 / 2}(-1)^{n}}{x^{1 / 4}(\nu-x)^{1 / 4}} \cos g+O\left[\frac{1}{(\nu x)^{3 / 4}}+\frac{\nu^{1 / 4}}{(\nu-x)^{7 / 4}}\right], \quad 1 \leqq x \leqq \nu-\nu^{1 / 3}
\end{aligned}
$$




$$
\mathscr{L}_{n+1}^{\alpha}(x)-\mathscr{L}_{n-1}^{\alpha}(x)=\frac{2^{5 / 2}(-1)^{n-1} x^{1 / 4}}{\pi^{1 / 2} \nu^{3 / 4}}\left(\sin g+O\left[\frac{x}{\nu}+\frac{1}{x}\right]\right), \quad 1 \leqq x \leqq \frac{1}{2} \nu .
$$

The derivations of (7.2)-(7.7) are as follows. (7.2) is (2.4) of [4] with the interval of validity reduced to simplify the statement of Lemmas 14 and 15. (7.3) is derived from (7.2); the only difficulty in doing this is the problem of obtaining suitable expressions for $g(N+2, x)-g(N, x)$ and $g(N-2, x)-g(N, x)$. For this, observe that $g_{N}(N, x)=-\frac{1}{2} \cos ^{-1}\left(x N^{-1 / 2}\right)$. Then using the fact that $\cos ^{-1}(y)$ $=\frac{1}{2} \pi-y+O\left(y^{3}\right)$ for $0 \leqq y \leqq 1$ and integrating shows that $g(N+2, x)-g(N, x)$ $=-\frac{1}{2} \pi+x N^{-1 / 2}+O\left(x^{3} N^{-3 / 2}\right)$ and $g(N, x)-g(N-2, x)$ has the same estimate. With this, (7.3) is easy to prove.

For (7.4), use is made of the fact obtained from (8.22.4), p. 197 of [5] that

$$
\mathscr{L}_{n}^{\alpha}(x)=J_{\alpha}\left[(\nu x)^{1 / 2}\right](1+O(1 / n))+O\left(\nu^{-3 / 4} x^{5 / 4}\right), \quad \frac{1}{\nu} \leqq x \leqq 1 .
$$

Using the estimate of the Bessel function

$$
J_{\alpha}(z)=(2 / \pi z)^{1 / 2} \cos (z-\alpha \pi / 2-\pi / 4)+O\left(z^{-3 / 2}\right), \quad z \geqq 1,
$$

(1.71.6), p. 15 of [5], in (7.8) immediately gives (7.4).

To prove (7.5), use (7.8) for $\mathscr{L}_{n+1}^{\alpha}(x)$ and $\mathscr{L}_{n-1}^{\alpha}(x)$. Now (2), p. 45 of [6] states that $J_{\alpha}^{\prime}(z)=\frac{1}{2}\left(J_{\alpha-1}(z)-J_{\alpha+1}(z)\right)$. This, the expression obtained from this for $J_{\alpha}^{\prime \prime}(z)$, Taylor's formula and the given estimate for $J_{\alpha}$ will give a sufficiently good estimate of the difference of the two Bessel functions that arise to produce (7.5).

Except for the case when $1 \leqq x \leqq \frac{1}{2} \nu$ and $-1<\alpha<0$, (7.6) can be obtained by combining (2.8) and (2.11) of [4]. If $1 \leqq x \leqq \frac{1}{2} \nu$ and $-1<\alpha<0$, observe that starting with (2.7) and using (2.5) it can be shown that

$$
\mathscr{L}_{n}^{\alpha}(x)=-\mathscr{L}_{n-2}^{\alpha+2}(x)-(4 x / v)^{1 / 2} \mathscr{L}_{n-1}^{\alpha+1}(x)+O\left[(n x)^{-3 / 4}\right] .
$$

Now let $p(x, n)$ be the principal term on the right side of (7.6). Substituting (7.6) into (7.9) and using this notation shows that for $\alpha \geqq 0$ and $1 \leqq x \leqq \frac{1}{2} \nu$

$$
p(x, v)=-p(x, v-4)+(4 x / v)^{1 / 2} p(x, \nu-2)+O\left[(\nu x)^{-3 / 4}\right] .
$$

Since $\alpha$ does not appear in (7.10), the condition that $\alpha>0$ is not necessary and (7.10) is true whenever $1 \leqq x \leqq \frac{1}{2} \nu$.

Now if $-1<\alpha<0$, replace the functions on the right side of (7.9) by the estimates in (7.6) and then use (7.10). This will produce (7.6) for the case $1 \leqq x \leqq \frac{1}{2} \nu$ and $-1<\alpha<0$.

(7.7) is derived from (7.6) in the same way that (7.3) was derived from (7.2).

Now the two following lemmas about (7.2)-(7.7) can be proved.

Lemma 14. Given one of the expressions (7.2)-(7.7), let $h(x)$ be the argument of its trigonometric function, $k(x)$ be the coefficient of this trigonometric function, and let $[s, t]$ be a subinterval of the stated interval of validity such that $h(t)-h(s)=2 \pi$. 
Then there exists $K$, independent of $s$ and $t$, such that if $u$ and $v$ are in $[s, t]$, then $t / s<K,|k(u) / k(v)|<K$ and $\left|h^{\prime}(u) / h^{\prime}(v)\right|<K$.

Lemma 14 need only be proved for (7.2), (7.4) and (7.6) since the result for the others follows immediately from the result for these. To prove it for (7.2), observe first that the partial derivative

$$
g_{2}(N, x)=\left(N-x^{2}\right)^{1 / 2} .
$$

Then write $t=N^{1 / 2}-N^{a}$; since $1 \leqq t \leqq N^{1 / 2}-N^{-1 / 6},-\frac{1}{6} \leqq a<\frac{1}{2}$. (7.11) shows that $g_{2}(N, x) \geqq N^{a / 2+1 / 4} \geqq N^{-a}$ for $x$ in $[s, t]$. By the law of the mean $(t-s) N^{-a}$ $\leqq g_{2}(N, t)-g_{2}(N, x)=2 \pi$ so that $s>N^{1 / 2}-8 N^{a}$. Then if $u$ is in $[s, t], N^{a / 2+1 / 4}$ $\leqq g_{2}(N, u) \leqq 4 N^{a / 2+1 / 4}$. This proves the last assertion for (7.2). The fact that $|k(u) / k(v)|<K$ follows immediately since $k$ is essentially the square root of $g_{2}$. If $t \geqq 9 N^{1 / 2} / 10$, then $s \geqq 2 N^{1 / 2} / 10$ and their ratio is bounded by 5 . If $t<9 N^{1 / 2} / 10$, then $g_{2}(N, u) \geqq 2 N^{1 / 2} / 5$ for $u$ in $[s, t]$ and $t-s<20$. Since $s \geqq 1$, this again gives a bound for $t / s$.

The proof of Lemma 14 for (7.4) is simple since $t=\left[(\nu s)^{1 / 2}+2 \pi\right]^{2} / \nu$. From this $t / s=\left[1+2 \pi(\nu s)^{-1 / 2}\right]^{2}$. Since $s \geqq 1 / v, t / s$ is bounded. The other inequalities follow immediately from this.

To prove Lemma 14 for (7.6) observe first that $h^{\prime}(x)=\frac{1}{2}(\nu-x)^{1 / 2} x^{-1 / 2}$. If $24 \nu / 25 \leqq t \leqq \nu-\nu^{1 / 3}$, write $t=\nu-\nu^{a}$. Then by the law of the mean $s \geqq \nu-14 \nu^{a}$. That $(\nu-s) /(\nu-t)$ is bounded is immediate and since $s \geqq 2 \nu / 5, t / s$ is also bounded. These facts prove the result in this case. If $1 \leqq t<24 \nu / 25$, then $h^{\prime}(u) \geqq \nu^{1 / 2} / 10 t^{1 / 2}$ for $u$ in $[s, t]$ and the law of the mean shows that $t \leqq s+70$. Since $s \geqq 1, t / s$ is bounded. That $(\nu-s) /(\nu-t)$ is bounded follows immediately from the fact that $t \leqq 24 \nu / 25$. These results prove the desired boundedness and complete the proof of Lemma 14.

LEMMA 15. Given one of the expressions (7.2)-(7.7) let $F(x)$ be the principal term, $k(x)$ be the coefficient of the trigonometric function in $F(x), K$ the constant of Lemma $14,[s, t]$ a subinterval of the stated interval of validity such that $t \geqq K s, 1 \leqq p<\infty$ and a real. Then there exists $C>0$, depending only on a and $p$, such that

$$
\int_{s}^{t}\left|F(x) x^{a}\right|^{p} d x \geqq C \int_{s}^{t}\left|k(x) x^{a}\right|^{p} d x .
$$

By inspection or by (7.11), the argument, $h(x)$, of the trigonometric function is monotone increasing. Then it is possible to partition $[s, t]$ into subintervals $S_{1}, S_{2}, \ldots, S_{j}$, so that on each, except possibly the first, $h(x)$ increases by $2 \pi$. On each $S_{i}$, Lemma 14 asserts that $h^{\prime}(x)$ varies by no more than a factor of $K$; therefore, for $i>1$ the trigonometric function has absolute value greater than $\frac{1}{2}$ on subintervals of $S_{i}$ with total length at least $2 /(K+2)$ times the length of $S_{i}$. Combining this with the fact, also from Lemma 14, that $x^{a} k(x)$ varies by no more than a fixed factor on $S_{i}$ shows that there is a constant, $C_{1}$, such that

$$
\sup _{x \in S_{i}}\left|k(x) x^{a}\right|^{p} \text { length }\left(S_{i}\right) \leqq C_{1} \int_{S_{i}}\left|F(x) x^{a}\right|^{p} d x
$$


for $1<i \leqq j$. This immediately implies that

$$
\int_{S_{i}}\left|k(x) x^{a}\right|^{p} d x \leqq C_{1} \int_{S_{i}}\left|F(x) x^{a}\right|^{p} d x, \quad 1<i \leqq j .
$$

Since $t \geqq K s$, by Lemma $14 h(t)>h(s)+2 \pi$ so that $j>1$. Since in each case $h^{\prime}(x)$ is a monotone decreasing function, $S_{1}$ is shorter than $S_{2}$. Using Lemma 14 again then shows that there is a constant, $C_{2}$, such that

$$
\int_{S_{1}}\left|k(x) x^{a}\right|^{p} d x \leqq C_{2} \int_{S_{2}}\left|k(x) x^{a}\right|^{p} d x
$$

Combining this with (7.12) for $i=2$ then shows that

$$
\int_{S_{1}}\left|k(x) x^{a}\right|^{p} d x \leqq C_{1} C_{2} \int_{S_{2}}\left|F(x) x^{a}\right|^{p} d x .
$$

Now add all the inequalities (7.12) together and add this sum to (7.13). Increasing the coefficient of each integral on the right to $C_{1}+C_{1} C_{2}$ and then dividing by this constant gives the conclusion of the lemma.

8. Proving the conditions are best possible. This section contains the proof of the following theorem.

THEOREM 15. If any of the inequalities for $a, A, b$ or $B$ in the hypotheses of Theorems 1-12 fails to hold, with the exception of equality in the strict inequalities for $B$ in the cases when $p=4 / 3$ or 4 and $\beta=1$, there is a function, $f$, for which the conclusion of the theorem is false. In the cases for which $\beta=1$, the theorem fails if $\beta$ is taken to be 0 .

The exception arises because the log term on the right side in those cases causes the standard counterexamples to fail. Theorems 1 and 7 are probably true with weak inequalities for $B$ when $\beta=1$, but to prove this seems more troublesome than is justified by the result. Theorem 15 does not give very precise information about the minimum possible value for $\beta$ in the cases where it was taken to be 1 . Here again a precise determination of this minimum seems more troublesome than is justified by its interest.

The proof of Theorem 15 as it refers to Theorem 7 will be given in detail since it includes most of the features of the other proofs. At the end a few comments will be made about how Theorem 15 is proved for the other theorems.

From Lemma 1 and the fact that for polynomials, $f(x), s_{n}(f, x)$ converges to $f(x)$ everywhere, it follows that $U(x)$ must be bounded by a constant times $V(x)$. From this it is immediate that $a \geqq A$ and $b \leqq B$ are necessary conditions for Theorem 7 to hold.

If the conclusion of Theorem 7 holds, then, as observed in the proof of Theorem $14,(6.5),(6.6)$ and (6.7) must be true. In particular $\left\|\mathscr{L}_{n}^{\alpha}(x) u(x)\right\|_{p}$ must exist. Using (6.12) then shows that $\int_{0}^{1} x^{(\alpha / 2+a) p} d x$ must exist. This immediately shows that $a>-\frac{1}{2} \alpha-1 / p$. Similarly, $\int_{0}^{1} x^{(\alpha / 2-A) q} d x$ must exist so that $A<1+\frac{1}{2} \alpha-1 / p$. 
Next, a lower bound is needed for $\left\|\mathscr{L}_{n}^{\alpha}(x) u(x)\right\|_{p}$. To obtain this use (7.6) in

$$
\int_{v / 2}^{v-s v^{1 / 3}}\left|\mathscr{L}_{n}^{\alpha}(x) u(x)\right|^{p} d x
$$

Given an $s$, if $n$ is sufficiently large, Lemma 15 will apply and give an estimate for the principal term. If $1<p \leqq 4$ and $s=1$, the integral of the error term has lower order of magnitude than the integral of the principal term. For $p \geqq 4$, the two integrals have the same order of magnitude but taking $s$ large enough will make the error integral less than half the principal integral. This computation shows that there is an $n_{0}$ and $C>0$ such that if $n>n_{0}$, then

$$
\begin{aligned}
\left(\left\|\mathscr{L}_{n}^{\alpha}(x) u(x)\right\|_{p}\right)^{p} & \geqq C \nu^{b p+1-p / 2}, & & 1 \leqq p<4, \\
& \geqq C \nu^{b p-1} \log \nu, & & p=4, \\
& \geqq C \nu^{b p-(p-1) / 3}, & & 4<p<\infty .
\end{aligned}
$$

Similarly

$$
\begin{array}{rlrl}
\left(\left\|\mathscr{L}_{n}^{\alpha}(x) / v(x)\right\|_{q}\right)^{p} & \geqq C \nu^{-B p-1 / 3}, & 1 \leqq p<4 / 3, \\
& \geqq C \nu^{-B p-p+1}(\log \nu)^{(1-4 \beta) / 3}, & p & =4 / 3, \\
& \geqq C \nu^{-B p-1+p / 2}(\log \nu)^{-p \beta}, & 4 / 3<p<\infty .
\end{array}
$$

These and (6.5) immediately show that the inequalities (5.3) are necessary and that $\beta$ cannot be taken as 0 when $B=b$ and $p=4 / 3$ or 4 . Furthermore, (6.6) and (6.7) show that the weak inequalities in (5.1) and (5.2) must be true. (6.7) also shows that $B \geqq \frac{1}{4}-1 / p$ is necessary; in case $\beta=1$ this weak inequality is all that was asserted in Theorem 15.

The provision that equality cannot occur in both (5.3) and (5.1) or (5.2) is also easy to prove necessary. If equality occurs in both (5.1) and (5.3), then $b=7 / 12-1 / 3 p, p>4$ and $B=3 / 4-1 / p$. It follows immediately from Lemma 15 and (6.20) that $\int_{1}^{v / 2}\left|\mathscr{L}_{n}^{\alpha}(x) / v(x)\right|^{q} d x$ is bounded below by a positive constant times $\nu^{-q / 4} \log \nu$ for $n$ larger than some $n_{0}$. Then using this to estimate $\left\|\mathscr{L}_{n}^{\alpha}(x) / v(x)\right\|_{q}$ and (8.2) for $\left\|\mathscr{L}_{n}^{\alpha}(x) u(x)\right\|_{p}$ shows that (6.5) is violated. Similarly, if equality holds in (5.2) and (5.3), estimate $\left\|\mathscr{L}_{n}^{\alpha}(x) u(x)\right\|_{p}$ by using (7.6) and Lemma 15 on $\int_{1}^{v / 2}\left|\mathscr{L}_{n}^{\alpha}(x) u(x)\right|^{p} d x$. Combining this and the estimate of $\left\|\mathscr{L}_{n}^{\alpha}(x) / v(x)\right\|_{q}$ from (8.3) produces a violation of (6.5).

All that remains of the proof that Theorem 15 applies to Theorem 7 is the proof of the necessity of the conditions $a>\frac{1}{4}-1 / p, A<3 / 4-1 / p, b<3 / 4-1 / p$ and, when $\beta=0, B>\frac{1}{4}-1 / p$. This part of the proof is slightly harder since these conditions are not necessary to make (6.5) hold. For these inequalities it will be shown directly that if equality occurs in any of them, then there is a counterexample to the conclusion of Theorem 7. This is sufficient, for if that conclusion fails for a particular $a$ or $B$, it fails for all smaller $a$ or $B$, and if it fails for a particular $A$ or $b$, it fails for all larger $A$ or $b$. 
If $a=\frac{1}{4}-1 / p$, let $K$ be the constant of Lemma 14. Choose $s$ so that $K s \leqq 1$ and so that for $n$ sufficiently large $\int_{s}^{K s}\left|\mathscr{L}_{n+1}^{\alpha}(x)-\mathscr{L}_{n-1}^{\alpha}(x)\right| d x$, when estimated by use of (7.5) and Lemma 15, will have its principal term at least twice its error term. Let $g_{n}(x)=\operatorname{sgn}\left(\mathscr{L}_{n+1}^{\alpha}(x)-\mathscr{L}_{n-1}^{\alpha}(x)\right)$ on $[s, K s]$ and 0 elsewhere. Then there exists $C>0$ and $n_{0}$ such that if $n>n_{0}$,

$$
\int_{s}^{K s} g_{n}(y)\left(\mathscr{L}_{n+1}^{\alpha}(y)-\mathscr{L}_{n-1}^{\alpha}(y)\right) d y \geqq C \nu^{-3 / 4} .
$$

If the conclusion of Theorem 7 were true for $a=\frac{1}{4}-1 / p$, there would be a $C$ independent of $n$ such that

$$
\int_{1 / v}^{s / 2}\left|\int_{s}^{K s} g_{n}(y) D_{n}(x, y) x^{a} d y\right|^{p} d x \leqq C .
$$

It will be shown that

$$
\int_{1 / \nu}^{s / 2}\left|\int_{s}^{K s} g_{n}(y) j_{i}(n, x, y) x^{a} d y\right|^{p} d x
$$

is a bounded function of $n$ for $i=1$ and 2 but is not a bounded function of $n$ for $i=3$. Because of (2.22) and the fact that the $c_{n}$ in (2.22) is bounded below by a positive constant, these facts concerning (8.6) will be sufficient to show that (8.5) cannot be true.

To show that for $i=1(8.6)$ is a bounded function of $n$ is a simple matter of using the value of $j_{1},(2.18)$, and the estimates (2.5). That (8.6) is bounded for $i=2$ is also simple since the $x-y$ term in $j_{2}$ can be replaced by $s / 2$, the estimates (2.5) and (2.6) used and the integrals computed. For $i=3,(8.6)$ is bounded below by

$$
\left(\int_{s}^{K s} \frac{n}{K s}\left|\mathscr{L}_{n+1}^{\alpha}(y)-\mathscr{L}_{n-1}^{\alpha}(y)\right| d y\right)^{p} \int_{1 / v}^{s / 2}\left|\mathscr{L}_{n}^{\alpha}(x) x^{a}\right|^{p} d x .
$$

If $n$ is sufficiently large, $K / \nu \leqq \frac{1}{2} s$ and Lemma 15 can be applied to both integrals in (8.7). Using (7.5) and (8.2) gives a lower bound of $C \log \nu$ for (8.7) for $n$ sufficiently large. This completes the proof that $a$ cannot equal $\frac{1}{4}-1 / p$.

If $A=3 / 4-1 / p$, fix $a$ and choose $s$ so that $K s \leqq 1$ and so that for sufficiently large $n \int_{s}^{K s} x^{a p}\left|\mathscr{L}_{n+1}^{\alpha}(x)-\mathscr{L}_{n-1}^{\alpha}(x)\right|^{p} d x$, when estimated by use of (7.5) and Lemma 15 , has its principal term at least twice the error term. Let $g_{n}(x)=x^{-3 / 4} \operatorname{sgn} \mathscr{L}_{n}^{\alpha}(x)$ on $\left[1 / \nu, \frac{1}{2} s\right]$ and 0 elsewhere. Then if the conclusion of Theorem 7 were true, there would be a $C$ such that

$$
\int_{s}^{K s}\left|\int_{1 / v}^{s / 2} g_{n}(y) D_{n}(x, y) x^{a} d y\right|^{p} d x \leqq C \log v .
$$

If $D_{n}$ is replaced by $j_{1}$ or $j_{3}$ in (8.8), it is easy to see that the inequality holds. The left side of (8.8) with $D_{n}$ replaced by $j_{2}$ is bounded below by

$$
\int_{s}^{K s} x^{a p}\left|\mathscr{L}_{n+1}^{\alpha}(x)-\mathscr{L}_{n-1}^{\alpha}(x)\right|^{p} d x\left(\int_{1 / v}^{s / 2} \frac{n}{K s}\left|\mathscr{L}_{n}^{\alpha}(y)\right| y^{-3 / 4} d y\right)^{p}
$$


For $n$ sufficiently large, (7.5), (7.6), Lemma 15 and the definition of $s$ show that the first integral in (8.9) is bounded below by a positive constant times $\nu^{-3 p / 4}$ and the second is bounded below by a positive constant times $\nu^{3 p / 4}(\log \nu)^{p}$. Therefore, (8.9) is not bounded above by the right side of (8.8); as in the last case this is sufficient to show that (8.8) cannot be true.

If $b=3 / 4-1 / p$, choose $s>1$ so that the integral of $\left|\mathscr{L}_{n}^{\alpha}(x)\right|$ over $[s, K s]$ is greater than a constant times $\nu^{-1 / 4}$ for $n$ large; this is possible by (7.6) and Lemma 15. Let $g_{n}(y)=\operatorname{sgn} \mathscr{L}_{n}^{\alpha}(y)$ on $[s, K s]$ and 0 elsewhere. Then the conclusion of Theorem 7 implies that

$$
\int_{2 K s}^{v / 2}\left|\int_{s}^{K s} g_{n}(y) D_{n}(x, y) d y\right|^{p} d x \leqq C .
$$

The proof that this is impossible is like the two previous proofs; in this case it is $j_{2}$ that causes the difficulties.

Similarly, if $B=\frac{1}{4}-1 / p$, fix $b$ and choose $s>1$ so that the integral of $x^{b p}\left|\mathscr{L}_{n}^{\alpha}(x)\right|^{p}$ on $[s, K s]$ is, for large $n$, greater than a constant times $\nu^{-p / 4}$. Let

$$
g_{n}(y)=y^{-1 / 4} \operatorname{sgn}\left[\mathscr{L}_{n+1}^{\alpha}(y)-\mathscr{L}_{n-1}^{\alpha}(y)\right] .
$$

In this case the conclusion of Theorem 7 requires that

$$
\int_{s}^{K s}\left|\int_{2 K s}^{\nu / 2} g_{n}(y) D_{n}(x, y) d y\right|^{p} d x \leqq C \log \nu .
$$

Again, it is easy to show that this is impossible; here it is $j_{3}$ that causes the trouble.

This completes the proof of Theorem 15 as it applies to Theorem 7 .

The proof that Theorem 15 applies to Theorem 8 is somewhat different since (6.5) cannot be used. A replacement could be derived, but it is simpler to use the fact that if Theorem 8 holds, then the $L^{1}$ norm of the individual terms are bounded by the right side of the conclusion of Theorem 8 . Consequently, if $U$ and $V$ satisfy the conclusion of Theorem 8 and $u$ and $v$ are as before, there is a $C$, independent of $g$, such that

$$
\begin{aligned}
\left|\int_{0}^{\infty} g(y) \mathscr{L}_{n}^{\alpha}(y) d y\right| \int_{0}^{\infty}\left|\mathscr{L}_{n}^{\alpha}(x)\right| u(x) d x \\
\qquad C+C \int_{0}^{\infty}|g(x)| v(x)\left(1+\log ^{+} x+\log ^{+}|g(x)|\right) d x .
\end{aligned}
$$

Since the second integral on the left side of (8.10) must be finite, (6.12) shows that $a>-1-\frac{1}{2} \alpha$. If $A>\frac{1}{2} \alpha, g$ could be taken equal to $x^{-A / 2+\alpha / 4-1}$ on $[0,1]$ and 0 elsewhere. Then (6.12) shows that the first integral on the left side of (8.10) is infinite while the right side of (8.10) is finite; this contradiction shows that $A \leqq \frac{1}{2} \alpha$.

Now, given an $n$, let $g(x)=1 /(\nu-x)$ on $\left[\frac{1}{2} \nu, \nu-\nu^{1 / 3}\right]$. Then the first integral on the left side of (8.10) is bounded below by a constant times $\nu^{-1 / 3}$ by use of (7.4) and the obvious modification of Lemma 15 to permit $g(x)$ in the integrand. The second integral on the left side of (8.10) is bounded below by a constant times 
$\min \left(\nu^{-1 / 4}, \nu^{b+1 / 2}\right)$ by use of (8.2), (7.4) and Lemma 15. The right side of (8.10) is bounded above by a constant times $\nu^{B}(\log \nu)^{2}$. Combining these facts shows that $b \leqq B-1 / 6$ and $B \geqq-7 / 12$.

That $A \leqq-\frac{1}{4}$ is necessary is proved with the same example and technique that showed $A=3 / 4-1 / p$ was impossible for Theorem 7 . The necessity of the other inequalities is proved in exactly the same way that the corresponding ones were proved necessary for Theorem 7 .

For Theorems 9 and 10 it is easy to derive an analogue of (6.5). This is done by using the converse of Hölder's inequality on the fact that the appropriate integral of the individual terms is bounded by the right side of the conclusion. The necessity of the conditions for these theorems is then proved in the same way that the corresponding conditions were treated for Theorem 7. The proof of Theorem 15 for Theorem 11 is like the proof for Theorem 8 . The application of Theorem 15 to Theorem 12 follows immediately from the preceding and the Banach Steinhaus theorem.

The proof that Theorem 15 applies to Theorems $1-6$ is similar to the preceding but somewhat simpler.

\section{REFERENCES}

1. R. Askey and S. Wainger, Mean convergence of expansions in Laguerre and Hermite series, Amer. J. Math. 87 (1965), 695-708. MR 32 \#316.

2. A. P. Calderon and A. Zygmund, On the existence of certain singular integrals, Acta Math. 88 (1952), 85-139. MR 14, 637.

3. B. Muckenhoupt, Hermite conjugate expansions, Trans. Amer. Math. Soc. 139 (1969), 243-260.

4. - Mean convergence of Hermite and Laguerre series. I, Trans. Amer. Math. Soc. 147 (1970), 419-431.

5. G. Szegö, Orthogonal polynomials, rev. ed., Amer. Math. Soc. Colloq. Publ., vol. 23, Amer. Math. Soc., Providence, R. I., 1959. MR 21 \#5029.

6. G. Watson, A treatise on the theory of Bessel functions, Cambridge Univ. Press, Cambridge, 1966.

7. A. Zygmund, Trigonometric series, Vols. I, II, 2nd rev. ed., Cambridge Univ. Press, New York, 1959. MR 21 \#6498.

8. G. H. Hardy and J. E. Littlewood, Some more theorems concerning Fourier series and Fourier power series, Duke Math. J. 2 (1936), 354-382.

RUTGERS UNIVERSITY, New Brunswick, New Jersey

THe Institute For AdVANCEd Study, Princeton, New Jersey 